\title{
XI.
}

\section{Ueber congenitale Lage- und Bildungsanomalien der Nieren.}

(Aus dem Pathologischen Institut zu Heidelberg.)

Von Dr. Georg Strube

aus Bremen.

Abnormitäten in der Zahl und Lage der Nieren gehören nicht zu den seltensten pathologischen Befunden und sind dem beobachtenden Anatomen seit Alters bekannt. Die häufigste solcher Anomalien, der einseitige Nierendefect, findet sich schon bei Aristoteles erwähnt und ist als ziemlich gewöhnliches Vorkommniss bis in die neueste Zeit wieder und wieder geschildert und in seinen praktischen Consequenzen auf's Eingehendste gewürdigt worden. - Auch über die Anomalien, mit denen sich diese Arbeit beschäftigt, über die congenitale Tieflage der Nieren, mit oder ohne Verwachsung der beiderseitigen Organe, findet man eine grosse Anzahl von Beobachtungen in der medicinischen Literatur der letzten vier Jahrhunderte beschrieben. Die auffällige Situsanomalie eines so beträchtlichen Organs, wie der Niere, sowie auch die gleichzeitig dabei vorhandenen Gefässvarietäten und sonstigen Situsveränderungen, entgingen natürlich der Beobachtung nicht, nachdem man angefangen hatte, regelmässig Sectionen auszuäben, und es ist kaum einer der nambaften Anatomen des 16. Jahrhunderts zu nennen, der nicht von Lageund Bildungsanomalien der Niere berichtet, sie mehr oder minder ausführlich beschreibt oder bildlich fixirt.

Die folgenden Jahrhunderte häuften die Beobachtungen dieser Art, die Beschreibung der einzelnen Fälle wurde immer ausführlicher und präciser und Reflexionen und Untersuchungen über die Entwickelungsgeschichte dieser Anomalien kamen hinzu.

Immer aber behielten die Veröffentlichungen den Charakter von Beiträgen zur speciellen pathologischen Anatomie, die für 
den wissenschaftlichen Forscher nicht ohne Interesse waren, für den praktischen Mediciner jedoch keine Bedeutung gewinnen konnten. Denn die Lageanomalie wurde immer zufällig auf dem Secirtisch entdeckt, sie hatte im Leben keine Erscheinungen gemacht und sich deshalb der Beobachtung des Arztes nicht aufgedrängt. -

In der vorliegenden Arbeit wird diejenige Lageanomalie der Niere, die man als Wanderniere bezeichnet, and die ja in hohem Grade das Interesse des praktischen Mediciners erregt, nicht berücksichtigt werden. Man kann die Lageanomalie, um die es sich bei der Wanderniere handelt, als secundäre bezeichnen: die Niere rückt von ihrer normalen Stelle nach vorn und unten. Im Gegensatz daza sind die Lageanomalien, die ich besprechen werde, primär: die Niere erreicht den Platz nie, den sie normaler Weise einnehmen soll, sondern sie wird an einer tiefer gelegenen Stelle des Abdomens dauernd fixirt. Auch die in dieser Arbeit geschilderten Fälle beanspruchen wesentlich als pathologisch-anatomische Befunde Interesse, sie vermehren die Zahl der früheren Beobachtungen, und wenn sie denselben auch in den Thatsachen und in der Auffassung nichts wesentlich Neues hinzufügen, so stellen doch die Nierenanomalien an sich, und die Complication derselben mit Missbildungen anderer Art, einen recht interessanten Befund dar.

Zwei der zu beschreibenden Fälle haben dagegen eine weitergehende Bedeutung und wenden sich nicht nur an den Anatomen, sondern beanspruchen in hohem Maasse die Aufmerksamkeit des praktischen Mediciners. Beide Fälle kamen in ärztliche Beobachtung und Behandlung, ehe sie zur anatomischen Untersuchung gelangten; bei beiden fand eine Autopsia in vivo statt und in den Schwierigkeiten und dem Ausgang der Operation trat die Bedeutung, welche solch eine Situsanomalie für den praktischen Mediciner gewinnen kann, deutlichst in die Erscheinung, vielleicht zum ersten Mal in so augenfälliger Weise. Aus früherer Zeit giebt es nur sehr wenige and weniger bedeutsame Erfahrungen dieser Art, aber der Fortschritt der modernen Chirurgie wird es unvermeidlich mit sich bringen, dass sie noch öfters gemacht werden, und es ist deshalb für den Chirurgen von Wichtigkeit, von den Lageanomalien der Niere und in 
Sonderheit von deren praktischen Consequenzen Einsicht zu nehmen. Auch dazu soll diese Arbeit einen Beitrag liefern.

Zum Schluss der Arbeit habe ich die Literatur über Bildungs- und Lageanomalien der Nieren, die ich ziemlich vollständig durchgesehen habe, zusammengestellt, da das bisher noch nicht in solcher Ausführlichkeit geschehen ist.

Die 4 Fälle, die ich zu beschreiben habe, kamen im Lauf der letzten drei Jahre im pathologisch-anatomischen Institut in Heidelberg zur Beobachtung und wurden mir von Herrn Geheimrath Arnold zur Bearbeitung überwiesen. Zwei von ihnen waren als zufälliger Sectionsbefund entdeckt worden, die beiden anderen waren vorber in der Heidelberger chirurgischen Klinik in Beobachtung und Behandlung gewesen. Ich erhielt von Herrn Geheimrath $\mathrm{Czerny}$ freundlichst die Erlaubniss die Kranken- und Operationsprotocolle dieser Fälle verwerthen zu dürfen.

Fall I.

Tieflage der linken Niere bei normaler Lage der rechten.

Das Kind M ... R ... E .,, 27 Tage alt, gestorben am 17. Juni 1891.

Bei der Section zeigte sich folgendes Verhalten der Nieren:

Die rechte Niere liegt an normaler Stelle, überlagert von der rechten Nebenniere. Auch die linke Nebenniere liegt an normaler Stelle; sie ist etwas grösser als die rechte. Die rechte Nebenniere ist $2 \frac{1}{4} \mathrm{~cm}$ lang, $2 \frac{3}{4}$ breit, die linke ist $3 \frac{1}{2} \mathrm{~cm}$ lang, $2 \frac{1}{2} \mathrm{~cm}$ breit.

Die linke Niere liegt tief, auf den letzten Lendenwirbeln, vor der Aortentheilung. Sie überragt die Mittellinie des Körpers nach rechts hin, gehört aber mit ihrem grösseren Theil der linken Fossa iliaca an. Ibr oberes Ende liegt $2 \frac{1}{2} \mathrm{~cm}$ oberhalb der Aortentheilung; nach abwärts ragt sie in's kleine Becken hinein, so dass ibr unterer Pol dem Fundus uteri aufliegt, und sie den Eingang in den binteren Douglas'schen Raum verschliesst. Zu beiden Seiten des unteren Nierenendes liegen die Ovarien.

An der Vorderfläcbe überzieht das Peritonäum die Niere.

Dieseibe stellt ein länglich rundes, von vorn nach hinten abgeplattetes Gebilde dar. Die hintere Fläche ist glatt, die vordere zeigt mehrere Furchen, welche vom Rande nach der Mitte zu verlaufen, welche den Hilus trägt.

Die Grössenverhältnisse sind:

\begin{tabular}{|c|c|c|c|}
\hline 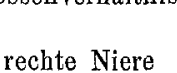 & . & $\begin{array}{l}\text { Länge. } \\
\text {. } 4 \frac{3}{4} \mathrm{~cm}\end{array}$ & $\begin{array}{l}\text { Breite. } \\
2 \frac{3}{4} \mathrm{~cm}\end{array}$ \\
\hline inke Niere & & $5 \frac{1}{4}-$ & \\
\hline
\end{tabular}

Die linke Niere ist also voluminöser als die rechte, indem sie dieselbe sowohl an Breite als an Länge übertrifft.

Das Nierenbecken der linken Niere ist ein doppeltes. Ein Becken geht aus dem oberen Theil des Hilus hervor, ein zweites aus dem unteren, ein

Archiv f. pathol. Anat. Bd. 137. Hft. 2. 
kurzer 落anal verbindet beide. Aus dem unteren Becken geht der Jreter hervor. Dieser verlänft an der linken Beckenwand entlang, um an normaler Stelle in die Blase zu münden. Auch der rechte Ureter roündet normal. Die Blase ist von normaler Grösse und Gestalt.

Die linke Niere wird von drei Arterien versorgt. Zwei davon gehen dicht oberhalb der Bifurcation ans der linken Bälfte der Aorta hervor, die dritte $2 \mathrm{~cm}$ weiter oberhalb. Alle 3 dringen an der Einterfläche in die Nierensubstanz ein,

Die Nierenvene geht aus der Vorderfläche der Niere hervor, läuft neben der Aorta in die Höhe und vereinigt sich mit der von der linken Nebenniere kommenden Vena suprarenalis. Die beideu Venen laufen in derselben

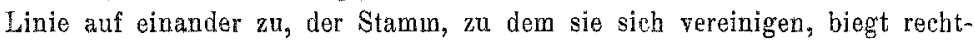
winklig gegen die bisherige Richtung ab und mündet in die Vena cava inferior.

Die rechte Niere wird von zwei Arterien versorgt: die Hauptarterie gebt an normaler Stelle aus der Aorta hervor und dringt am Hilus in die Niere ein. Ein zweiter Stamm entspringt in der Höbe der Arteria renalis sinistra superior aus der Aorta und tritt am unteren Pol in die Niere ein.

Die Vena renalis dextra kommt aus dem Hilus und mündet gegenüber der linken Vene in Vena cava. Etwas höher ergiesst sich in dieselbe die Vena suprarenalis dextra.

Der Tractus intestinalis und die Geschlechtsorgane zeigen weder Situsveränderungen, noch sonstige Anomalien.

Es handelte sich hier also um einen völlig uncomplicirten Fall von Tieflage der linken Niere, dessen anatomische Verhältnisse sebr einfache waren.

Fall 11 .

Tieflage der rechten Niere, Fehlen der linien, Atresia ani vesicalis ${ }^{1}$ ).

I. ... K . . . Kind männlichen Geschlechts, 4 Wochen alt, gestorben am 5. Februar 1893. Das Kind warde gleich nach der Geburt in die chirurgische Universitätsklinik gebracht, weil man das Feblen des Afters bemerkt hatte. Aus der Krankengesehichte sei Folgendes erwähnt:

Es bestebt eine stecknadelkopfgrosse Phimose. Aus der Urethra geht mit Koth vermengter Urin im Strahl ab. Die Analöffnung fehlt ganz. Der Leib ist aufgetrieben, tympanitisch schallend. Ein Längsschnitt vom Steissbein über das Perineum eröffuete das Cavum ischio-rectale, in welchem grösstentheils stumpf weiterpräparirt wurde. Das Rectum war trotz der vollständigen Blosslegung dieses Raumes nicht aufzufinden. Als nun das Steissbein an seiner Sacralverbindung durchtrennt und nach links berübergeklappt wurde, wölbte sich ein anscheinend mit Serosa überkleidetes, cylindrisches Organ vor, das als Darm imponirte. Dasselbe wurde mit zwei Fadenbändchen angeschlungen und mit dem Messer incidirt. Es entleerte

1) Der Fall ist kurz erwähnt in den Verhandlungen der deutschen Ges. f. Chirurgie, 22. Congress, 1893, S. 38 von Prof. Czerny. 
sich weder Koth noch eine Flüssigkeit. Die nähere Inspection ergab, dass ein parenchyma.toses Organ vorlag, vielleicht die Niero. Da das Feblen des eigentlichen Rectum damit festgestellt war, und man annehmen musste, dass der Darm direct in den Blasengrund münde, wurde die perineale Wundböble tamponirt und die Colostomia iliaca ausgeführt.

Das Kind erholte sich anfänglich ein wenig, zeigte Gewichtszunahme und die Wunden heilten gut. Doch fiel es bald ab, kam im Ernährungszustand sebr herunter und starb etwa 4 Wochen nach der Operation.

Der Sectionsbefund ergab:

An normaler Stelle findet sich keine der beiden Nieren. Die Nebennieren liegen als platte, ruadliche Gebilde von etwa Markstückgrösse zu beiden Seiten der Wirbelsäule in normaler Höhe. Die linke liegt etwas tiefer als die rechte.

Die einzige vorhandene Niere liegt tíef im kleinen Becken, dessen Böhle sie gänzlich ausfüllt, und an dessen Raumverhältnisse sie sich in ihrer Gestalt anpasst. Sie liegt mit ihrer Längsaxe genau in der Mittellinie des Körpers und stellt ein im Querschnitt viereckiges Gebilde dar, dessen Breite nach unten zu allmählich abnimmt, indem sich die Niere gleichsam zwischen die beiderseitigen Beckenwände einzwängt. Der untere Pol der Niere ruht auf den Weichtheilen des Beckenbodens. Nach oben reicht die Niere bis dicht unter die Ansatzstelle der Radix mesenterii. Die bintere Fläche der Niere ist convex gestaltet und rubt in der Wölbung des Kreuzbeins zwischen den beiderseits berabziehenden Psoasmuskeln. Die Aortentheilung liegt $1 \mathrm{~cm}$ tiefer als der obere Nierenpol. Die Niere drängt sich so zwischen die beiden Arteriae iliacae communes ein, dass dieselben, ein wenig nach aussen verlagert, auf den Seitenflächen der Niere beiderseits herab verlaufen.

Die Vorderfläche der Niere wird in ibrer oberen Partie vom Peritonäum überkleidet, das sieh in der rechten Körperhälfte auf die Blase überschlägt, eine Excavatio reno-vesicalis bildend. Links wird diese Bucht durch das sich zwischen Blase und Niere eindrängende Rectum noch in eine Excavatio vesico-rectalis und reno-rectalis getheilt. Die Vorderfläcbe ist durch Furchen in einzelne Abschnitte getheilt, platt in ibrew oberen Abschnitt, concav gewölbt im unteren, aus dem das Nierenbecken hervorgeht. Dasselbe entsteht aus vier Kelchen, von denen zwei aus der oberen, zwei aus der unteren Partie des Hilus kommen, und geht in einen $2 \frac{1}{2} \mathrm{~cm}$ langen, stellenweise bis $20 \frac{1}{2} \mathrm{~cm}$ breiten Ureter über, der sich in geschlängeltem Verlauf zur rechten Blasenhälfte begiebt nnd in dieselbe einmündet.

Die Länge der 'Niere beträgt $6 \mathrm{~cm}$; die Breite an der breitesten Stelle $3 \frac{1}{2}$, zwischen den Acetabulis gemessen $2 \frac{1}{2} \mathrm{~cm}$.

Die Niere erbält ihr Blut durch drei Arterien, die an der Vorderfläché in den Hilus eindringen, und zwar eine von rechts, die beiden anderen von links her.

Die Arteria renalis dextra entspringt $\frac{1}{2} \mathrm{~cm}$ oberbalb der Theilungsstelle der Aorta aus deren rechter Hälfte, verläuft erst an der Seitenfläche der Niere herab, wendet sich dann nach vorn und dringt in der Mitte der Vorder- 
fläche in den Hilus ein. Die beiden Arteriae renales sinistrae gehen aus einem kurzen gemeinsamen Stämmchen hervor, das aus der Arteria iliaca communis sinistra unmittelbar nach ibrem Abgang aus der Aorta entspringt. Der kurze, obere Ast verläuft direct nach abwärts und tritt im obersten Theil des Hilus in denselben ein. Der untere Ast dagegen läuft anf der Seitenfäche der Niere herab binter dem Rectum herum und dringt tiefer unten in den Hilus ein. An der Hinterfläche der Niere dringen linkerseits drei unbedentende Arterien, die aus Aorta, Iliaca comm. und lliaca interna sin. stammen, in die Nierenkapsel ein.

Die Venenstämmchen der rechten Seite, zwei ron der Vorderfläche, eine aus dem oberen Pol der Niere kommend, sammeln sich in eine gemeinsame Vene, die sich dicht oberbalb des Zusammenfusses der Venae iliacae in die Vena cava ascendeis ergiesst. Die Venen der linken Seite münden, gleichfalls in einem gemeinsamen Stamm, in die Vena iliaca externa der linken Seite.

Die grassen Venenstämme des Beckens zeigen ein von der Norm abweichendes Verbalten: Die Vena bypogastrica sinistra mündet nach rechts in die Vena iliaca communis dextra. Letztere vereinigt sich mit der Vena iliaca externa der linken Seite zur Caya ascendens.

In der linken Bauchseite, etwas einwärts von der Spina iliaca ant. sup., findet sich oberhalb des $\mathbf{S}$ romanum das Colon descendens in die Bauchwand eingewachsen, einen Anus praeternaturalis bildend. An der Stelle der Rimaani findet sich eine in der Richtung derselben verlaufende Narbe. Das Ende des Dickdarms schiebt sich, wie erwähnt, von links her zwischen Blase und Niere ein. Der Darm ist hier $2-2 \frac{1}{2} \mathrm{~cm}$ breit. Dann geht er plötzlich in einen $3 \mathrm{~mm}$ breiten und $1 \mathrm{~cm}$ langen Kanal über, der am Grund der Blase in der linken Seite einmündet, so dass man mit der Sonde vom Rectum aus in die Blase vordringen kann. Die beiden Samenbläschen überlagern die Einmündungsstelle des Darms in die Blase. An dieser Stelle treflen auch die beiden Vasa deferentia zusammen. Das rechte hat einen langen Weg an der hinteren Blasenwand oberhalb der Ureterenmündung zurüekgelegt, während das linke, von kurzem Verlauf, unter dem Rectum her sich zur Einmündungsstelle in den Sinus urogenitalis begiebt.

Die Blaso ist, wie schon aus dem soeben geschilderten Verlauf der Vasa deferentia hervorgeht, asymmetrisch gebaut, indem die rechte Hälfte stärker ausgebuchtet ist, als die linke. Die Blase ist stark ausgedehnt und läuft nach oben $z u$ in ein starkes Ligamentum vesico-umbilicale medium aus. Im Innern tritt die Asymmetrie ebenfalls deutlich hervor. Nur die rechte 
Ureterenmündung und der rechte Schenkel des Trigonum Lieutandi sind ausgebildet, links ist keine Spur davon nachweisbar. Dicht oberhalb des. Caput gallinaginis ist die Oeffnung, durch welche sich der Canalis recto-vesicalis in die Blase öffnet.

Die beiden folgenden schematischen Zeichnungen geben ein Bild der Lagebeziehungen von Niere, Rectum und Blase zu einander und von den anatomischen Verhältnissen, die sich an der hinteren Blasenwand vorfanden.

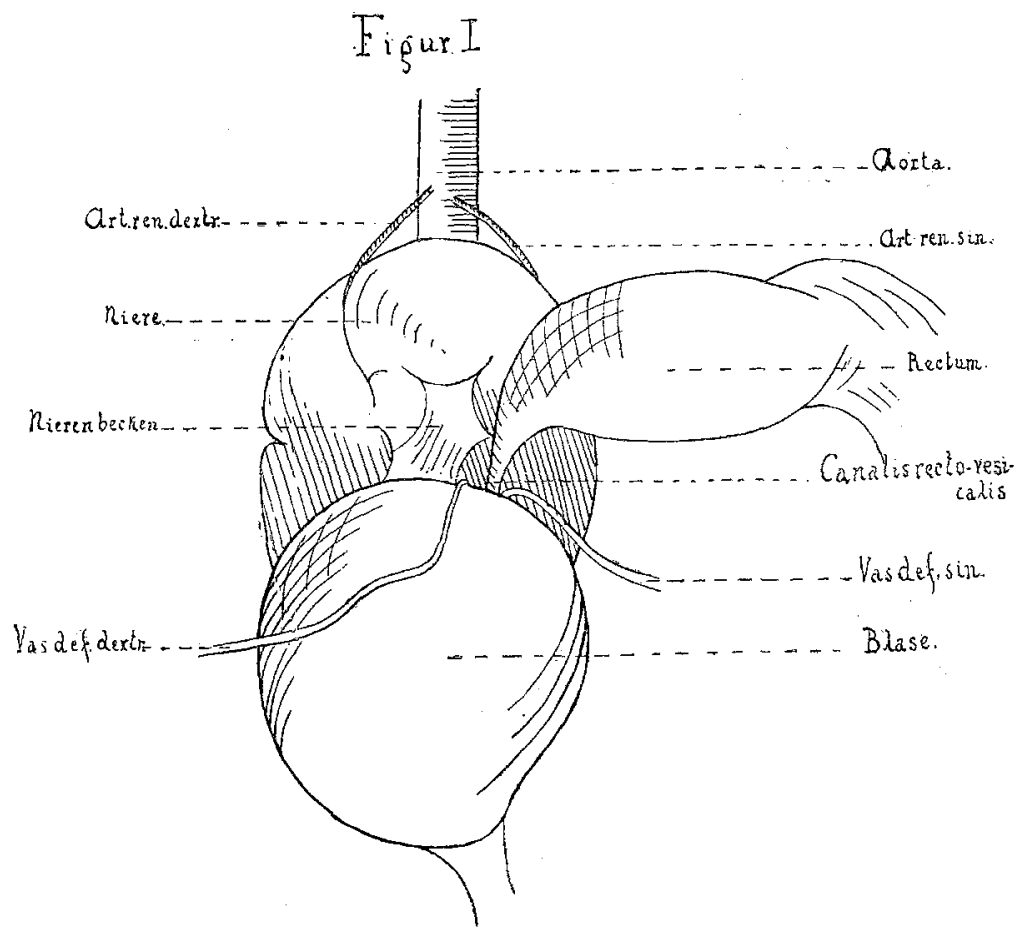

Figur I stellt die Niere in situ dar. Dieselbe bedeckt die Aorta bis oberhalb der Bifurcation. Aus der Aorta gehen 2 Nierenarterien hervor. Die Blase, welche bei dieser Ansicht von vorn die Niere völlig bedecken würde, ist nach vorn herunter geschlagen, so dass man in der Abbildung auf die Hinterwand der Blase sieht, über welche das rechte Vas deferens hinwegzieht. Von links her schiebt sich zwischen Niere und Blase das Rectum ein, welches, sich plötzlich verjüngend, in den in die Blase mündenden Kanal übergèt. Das linke Vas deferens hat nur einen kurzen Verlauf an der hinteren Blasenwand. In dem Verbalten der beiden Vasa deferentia tritt die Asymmetrie der beiden

Blasenhälften hervor. 


$$
\text { Figur } \mathbb{I}
$$

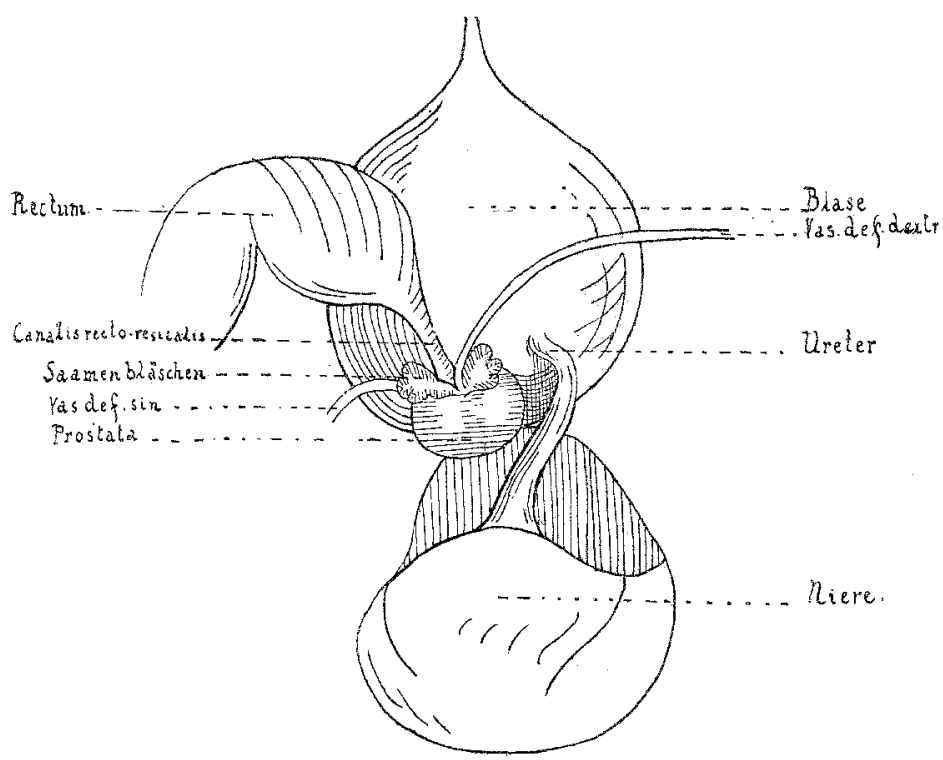

In Figur ll sieht man auf die hintere Blasenwand. Um diese zur Anschauung zu bringen, wurde die Niere, die hinter der Blase lag, völlig herunter geschlagen. Man blickt also auf die obere und vordere Fläche der Niere, aus letzterer sieht man den Ureter hervorgehen und rechterseits in die Blase münden. In der Lage der Prostata und der Samenbläschen, sowie in dem Verhalten des Ureters zur hinteren Blasenwand zeigt sich deutlicher als in der ersten Figur die Asymmetrie der Blasenbälften.

Der Fall ist nicht so einfach, wie der vorige: der Grad der Tieflage der Niere ist ein sehr beträchtlicher, es ist ein sehr ungewöhnlicher Befund, dass die Niere ganz in der kleinen Beckenhöhle liegt. Ferner handelte es sich bei diesem Falle um ein Fehlen der linken Niere und zwar um eine Agenesie derselben, wie aus dem Mangel des Ureters und der Asymmetrie der Blase hervorgeht. Am meisten Interesse erweckt der Fall jedoch durch die Complication mit Atresia ani vesicalis, einer Form der Atresie, die als persistirende Cloakenbildung aufgefasst werden muss. Das Zusammentreffen von Nierendystopie und Atresia ani ist ein sehr seltenes Vorkommniss. Es wird weiter unten zu erörtern sein, $a b$ es sich dabei um eine zufällige 
Coincidenz der beiden Missbildungen handelt, oder ob eine durch die andere verursacht worden ist. Praktisch ist von Interesse, dass die Unmöglichkeit àie Atresie durch eine Operation zu beseitigen durch die Tieflage der Niere hervorgerufen wurde.

Fall III.

Tieflage der linken Niere, Fehlen der rechten. Kryptorchismus rechtsseitig.

N..... H ... ans Oftersheim, 32 Jahre, gestorben 4. April 1891.

Aus der Krankengeschichte erwähne ich:

Anamnese: Der Patient leidet seit einem halben Jahre an hartnäckiger Verstopfung, sowie Beschwerden beim Wasserlassen, die als erschwertes Uriniren und Schmerzen in der Urethra zum Ausdruck kommen. Die Verstopfung weicht nur energischer Anwendung von Abführmitteln.

Der Koth besteht aus kleinen, harten, in schleimigen Eiter gehüllten Ballen; nur selten besteht normaler Stuhl.

Im Vorjahre soll der Unterleib geschwollen gewesen sein, was jedoch nach gründlicher Entleerung wieder verschwand. Ferner stellten sich morgendliches Erbrechen, Appetitlosigkeit, starker Zungenbelag, aufgeregte Stimmung, schlechter Schlaf ein, Symptome, die bis heute in wechselnder Stärke fortbestehen.

Anfang September vorigen Jahres wurde Patient gelb im Gesicht, gleichzeitig batte er Magensehmerzen, aber kein Erbrechen. Im Aussehen des Stuhls bemerkte er damals keine Veränderung. Die Gelbfärbung hielt bis in den October hinein an.

Im Jahr 1880 hatte Patient einen syphilitischen Primäraffect, und später secundäre Erscheinungen. Er machte eine Schmiercur durch. 1883 bekam er eine Gonorrhoe, die nach 3 Monaten ohne Complication heilte.

Patient giebt Potatorium zu. Seine seit 10 Jahren geführte Ehe ist steril.

Vor 4 Monaten wurde Patient von einem Arzte, den er wegen seiner Verstopfung consultirt hatte, auf einen Tumor im Unterleib aufmerksam gemach, der seiner Beachtung bisber entgangen war, so dass er über die Zeit und Art seiner Entstehung keine Angaben machen kann. Der Tumor soll damals dieselbe Grösse gehabt haben, wie heute, ist spontan nicht schmerzbaft. Bei starkem Druck auf den Tumor werden Schmerzen angegeben. Geringe Veränderungen in der Lage des Tumors glaubt Patient ofters zu bemerken, ohne dass die Füllung des Darms darauf von Einfuss wäre.

Status praesens: Ziemlich grosser, kräftig gebauter, muskelstarker Mann. Das Abdomen ist nicht aufgetrieben, die Bauchdecken sind straff. Die Leberdämpfung äberragt in der Parasternallinie den Rippensaum um 2 Querfinger. Die Milzdämpfung ist nicht vergrössert. Es bestehen kein Icterus und keine Oedeme. Bei tiefem Eindrücken fühlt man unterhalb des Nabels einen rundlichen Tumor, der auf Druck schmerzhaft ist. Die rechte Scrotalhälfte ist leer und auch im Leistenkanal ist der Hode nicht nach- 


\section{6}

weisbar, dagegen ist die Spertura externa des Leistentanals fine die Fingerkuppe durchgängig. Die Inguinaldrüsen sind beiderseits geschwollen.

Untersuchung in Narkose 6. Febr. 1891:

Etwa drei Finger breit unterhalb des Nabels, vor dem Promontorium, in's grosse Becken aufragend, etwas mehr naci rechts von der Mittellinie liegend, findet sich ein faustgrosser Tumor, dessen unterer Pol rom Rectum aus mit der Fingerspitze zu erreichen ist. Derselbe ist von etwa elliptischer Form, in querer Richtung etwas verschiebbar, in verticaler kaum. Er ist von uneben böckriger Oberfäche und weich elastischer Consistenz. In der Gegend des rechten Samenbläschens ist ein derberer Strang fühlbar, als auf der linken Seite.

Als klinische Diagnose wird mit Wahrscheinlichkeit: Sarcom des retinirten Hodens angenommen.

Am 1. April 1891 wurde die Laparotomie vorgenommen (Dr. v. Mejer).

Nach Eröffnung der Bauchböhle, durch einen Schnitt in der Linea alba. vom Nabel bis zur Symphyse, zeigt sich, dass der Tumor retroperitonäal liegt, und auf den ersten Blick imponirte die Geschwulst als ein retroperitonäales Lymphdrüsenpacket. Nach Eventeration der Därme und Einpacken derselben in Salicylcompressen, wird das Mesenterium in querer Richtung parallel dem Gefässverlauf durchtrennt und darauf stumpf auf den Tumor eingegangen. Derselbe hat eine rothbraune Farbe und scheint äusserlich otwa der Nierensubstanz zu entsprechen. Ein ungefähr wallnussgrosser Lappen wird freipräparirt, wobei eine grössere Anzahl grösserer und kleinerer arterieller und venöser Gefässe bluten und theils direct gefasst, theils schon vor der Durchtrennung ligirt werden. Der isolirte Lappen wird abgetrennt, wodurch eine ziemlich grosse parenchymatöse Blutung entsteht, welche durch Tamponade gestillt wird. Auf dem Durchschnitt zeigt sich der Lappen deutlich als Nierengewebe. Es liegt also jedenfalls eine Lageanomalie vor, vielleicht eine Fufeisenniere mit geringerer Entwickelung der rechten Hälfte. Eine einfache Wanderniere ist deshalb nicht wabrscheinlich, weil der Stiel sehr kurz war und die Niere sich sehr wenig bewegen liess. Yon dem Vorhandensein der rechten und linken Niere konnte man sich nicht bestimmt überzeugen. Auf die blutende Parenchymstelle wurden Jodoformdochte gelegt und nach aussen abgeleitet. Das Mesenterium wird durch eine fortlaufende Catgutnaht geschlossen und nach Zurückbringen der Eingeweide werden die Bauchdecken vereinigt und Verband augelegt.

2. April 1891. Nach der Operation trat kein Erbrechen ein. Die Schmerzen sind heftig und werden mit Morphium bekämpft. Nachss schlief der Patient wenig. Es trat einmal Erbrechen ein.

Urinmenge $275 \mathrm{cem}$. Spec. Gewicht 1025 .

Ilorgens beftige Schmerzen. Keine Bläbungen abgegangen. Der Leib ist ziemlich stark gespannt, kein Meteorismus. Starke Druckempfindlichkeit besonders links. Der Verband ist von blutigem Serum durchtränkt, nach Gintfernung der Dochte starker seröser Ausfluss aus der Drainageöfnung. Der Urin enthält viel Albumen und Hämoglobin. 
3. April. Urinmenge sehr gering. Auch beim Katheterisiren entleert sich kein Urin. In der linken Seite über dem Poupart'schen Band zeigt sich eine circumscripte Dämpfung, durch ein längeres Drainrohr, das eingeführt wird, entleert sich blutig-seröse Flässigkeit.

4. April. Vollkommene Anurie während 24 Stunden. Während der Nacht und Morgens beständiges Erbrechen. Um 8 Morgens trat der Tod ein.

Aus dem Sectionsprotocoll ist $z u$ berichten:

In dem peritonäalen Ueberzug der hinteren Bauchwand findet sich eine ausgebreitete hämorrbagische Infiltration, insbesondere in der linken Nierengegend. Entsprechend dem untersten Lenden- und obersten Kreuzbeinwirbel ist das Peritonäum. sehr stark vorgewölbt durch eine hinter demselben gelegene, etwa faustgrosse Geschwulstmasse. Auf der höchsten Stelle derselben ist das Peritonäum in einer Ausdehnung von $5 \mathrm{~cm}$ eingeschnitten und durch Nähte wieder vereinigt.

Der Tumor erweist sich als die nach unten verlagerte Niere. Beide Nebennieren sind von normaler Grösse und Gefässversorgung und normal gelagert. Die vorbandene Niere reicht vom 4. Lendenwirbelkörper bis zum 2. Kreuzbeinwirbel. Sie ist $12 \mathrm{~cm}$ lang, $8 \mathrm{~cm}$ breit, $4,5 \mathrm{~cm}$ dick. Dies massige Organ liegt in der linken Darmbeingrube und der linken Hälfte des kleinen Beckens, so dass die Vasa iliaca sinistra ganz davon bedeckt werden, während die rechten frei bleiben. Der obere Pol liegt etwa in der Höhe der Aortentbeilung. Die Hinterfläche der Niere ist glatt, sie zeigt einen Eindruck entsprechend dem Psoas, sowie eine dem Rand des kleinen Beckens entsprechende Furche. Anf der Vorderfläche trägt die Niere eine Anzahl knoiliger Erhebungen, die durch Furchen, in denen zum Theil Arterien verlaufen, von einander getrennt sind. Eine dieser Prominenzen ist durch die Operation entfernt, und man erblickt an ibrer Stelle eine verschorfte Wundfläche. Die Gestalt des ganzen Organs ist die eines platten, rundlichen Kuchens. Der Ililus gehört der Vorderfläche an, bier dringen die Gefässe ein, von hier geht der Ureter hervor. Das Nierenbecken entsteht durch das Zusammenfliessen von vier Kelchen, zwei kleineren und zwei grösseren; und zwar kommt aus dem oberen und unteren Abschnitt der Niere je ein grosser Kelch, wäbrend die kleineren aus der Mitte der Vorderfläche hervorgeben. Das Nierenbecken zeigt in seinem obersten Abschnitt eine Continuitätstrennung, die durch Nähte vereinigt ist. Gleich darüber befindet sich die oben erwähnte Verletzung der Nierensubstanz. Der Ureter, der aus dem Nierenbecken hervorgeht, ist $11 \mathrm{~cm}$ lang, und sehr weit; er verläuft hinter dem Rectum herum, an der linken Beckenwand zur Blase herab, in die er sich in der linken Seite einsenkt. Am rechten Seitenrand der Niere ist ein Stück von bohnenförmiger Gestalt, etwa $4 \mathrm{~cm}$ lang, 2-3 $\mathrm{cm}$ breit, mit der Kapsel fest verwachsen und von derber, schwieliger Beschaffenheit. Die mikroskopisehe Untersuchung ergab, dass dies Stäck aus Resten von Glomerulis, mit colloider Masse erfüllten Harnkanälchen, und dickwandigen Gefässen besteht, also obliterirte Nierensubstanz darstellt.

Das bezeichnete Stück ist an der Vorderfläche durch eine Furche von 
der übrigen Nierenmasse abgegrenzt, während es hinten und am oberen Rande ohne Grenze in dieselbe übergeht. In der vorderen Grenzfurche $z$ wischen normalem und obliterirtem Nierengewebe verläuft ein Ureter, der zum grössten Theil vollständig obliterirt, an anderen Stellen, von Septen, Membranen und Filamenten durchzogen, für die Sonde mit Mühe durchgängig ist. Von diesem Ureter gehen strangförmige Fortsätze ab, sowobl nach rechts in das narbige Nierengewebe, als auch nach links in die normale Substanz. Nach oben erstreckt sich der obliterirte Ureter bis uber die Grenze des narbigen Theiles der Niere fort und sein oberes Ende steht scheinbar mit dem normal gebildeten Harn]eiter der linken Seite, bezw. mit dem Nierenbecken in Verbindung, denn man kann rom Nierenbecken ans mit der Sonde bis zu diesem obersten Abschnitt des obliterirten Elarnleiters vordringen, eine directe Communication aber nicht nachweisen, da die Obliteration an dieser Stelle eine besonders hochgradige ist. Der obliterirte Ureter mündet gleichfalls in die Blase, von wo aus er eine Strecke weit zu sondiren ist. Die Mündungsstelle findet sich an der hinteren Blasenwand rechts von der Mündung des normalen Harnleiters. Während aber von letzterem der Schenkel des Trigonum Lieutandi und der Ureterenwulst wohl ausgeprägt sind, fehlen dieselben bei dem obliterirten rechten Harnleiter, so dass der innere Bau der Blase trotz der doppelten Ureterenmündung asymmetrisch ist.

Die Gefässe der Niere treten sämmtlich am Hilus ein: es sind zwei Arterien, die von eben so viel Venen begleitet werden. Die Arteria renalis superior gebt $1 \frac{1}{2} \mathrm{~cm}$ oberhalb der Bifurcation aus der Aorta hervor, wendet sich zur rechten und oberen Partie der Niere und versorgt dieselbe durch zwei Aeste. Die letzteren verlaufen in den Furchen der Vorderfäche, um sich am Hilus in die Niere einzusenken. Die Arteria renalis superior ist aw oberen Rande der Niere ligirt. Die Venen dieses Nierenabschnittes verlaufen in Begleitung der Arterien, sammeln sich zu einem gemeinsamen Stamme, der in die Vena cava inferior einmindet.

Die Arteria renalis inferior gebt aus der Art. hypogastrica sinistra hervor, an einer Stelle, wo diese dem linken Nierenrande aufliegt. Die Arterie wendet sich direct zum Nierenhilus, in den sie eindringt. Beide Arterien sind von annähernd gleicher Stärke. Die Vena renalis inferior verläuft in Begleitung der Arterien und mündet in die Vena iliaca communis sinistra.

Auch in diesem Falle fand sich eine Anomalie der Venenverzweigung im Becken. Die linke Vena hypogastrica gabelt sich in zwei Aeste, von denen sich der schwächere mit der Vena iliaca externa zur Iliaca communis sinistra vereinigt, während der stärkere Ast in die Vena hypogastrica dextra einmündet.

Das Peritonäum überkleidet die Niere an der Vorderfiäche und schlägt sich dann auf Blase und Mastdarm über, indem letzterer sich von links her zwischen Blase und Niere einschiebt.

Vom rechten Hoden findet sich im Sectionsprotocoll berichtet, dass er im Leistenkanal lag, atrophisch war und mit dem Processus vaginalis ver- 
wachsen. Da die Hoden und Samenleiter an dem Präparat, das mir vorlag, nicht mehr vorhanden sind, kann über das Verhalten der Geschlechtsausfäbrwege, Vasa deferentia und Samenbläschen, welche Anomalien aufzuweisen schienen, nichts Genaues ausgesagt werden.

Bei diesem Falle war die Entscheidung der Frage, ob es sich um eine einfache tiefliegende oder um eine verwachsene Niere handle, nicht ganz leicht zu treffen. Wegen des Vorhandenseins von zwei Ureteren lag es nahe, das obliterirte Stück Nierengewebe als die rechte Niere anzusehen, welche mit der linken verwachsen und in der Entwickelung zurückgeblieben war, unter Obliteration ihres Ureters. Gegen diese Auffassung lassen sich jedoch folgende Punkte anführen. Erstens ist keine scharfe Grenze zwischen dem schwielig degenerirten und dem normalen Gewebe vorbanden, vielmehr ist der Uebergang ein ganz allmählicher, keine Grenzfurchen trennen, wie es in solchen Fällen stets gefunden wird, eine ursprünglich rechte und linke Niere. Namentlich ist keine Andeutung von einer selbständigen Kapselbildung nachweisbar. Zweitens setzt sich der obliterirte Ureter über die Grenzen des schwieligen Nierengewebes nach oben fort, er entsendet seine strangförmigen 'Fortsätze auch in die normale Niere, und steht, wie oben geschildert, mit dem normal gebildeten Harnleiter in Verbindung. Endlich spricht das Fehlen des rechten Schenkels des Trigonum Lieutandi und des rechten Ureterenwulstes gegen die Annahme, dass der obliterirte Harnleiter der der rechten Körperhälfte angehörige sei. Vielmehr scheint aus allen diesen Gründen die Auffassung sachgemässer, dass es sich um eine Niere handelt mit doppelter Ureterenbildung, und dass von diesen beiden Ureteren einer mit dem Stück der Niere, aus dem er hauptsächlich hervorgeht, obliterirt ist. Demnach schliesst sich dieser Fall an den vorigen an, mit dem er in einigen Punkten Uebereinstimmung zeigt.

Es handelt sich wieder um die Tieflage einer Niere, bei Feblen der anderen, nur ist der Grad der Tieflage nicht so beträchtlich, wie in jenem Fall. Als Besonderheiten verdienen hervorgehoben zu werden, erstens die beträchtliche Grösse des tiefliegenden Organs, die man als compensatorische Hypertrophie zu deuten hat, analog dem Hypervolum, das man bei einseitigem Nierendefect so häufig an dem restirenden Organ beobachtet hat. 
$Z_{\text {weitens ist das }}$ Zusammentreffen von Nierendystopie mit einem Bildungsfehler der Geschlechtsorgane bemerkenswerth; auf die Beziebungen zwischen Missbildungen der Niere und Sexualorgane wird weiter unten eingegangen werden. Besonderes Interesse hat der Fall aber durch die praktische Bedeutung, die er gewonnen hat, indem er zu den seltenen Fällen gehört, wo der Arzt auf den von der Niere gebildeten Tumor aufmerksam wurde. Durch das ungewöhnliche Zusammentreffen von Kryptorchismus und Nierendystopie wurde die äratliche Diagnose des Leidens irre geleitet. Die Operation klärte den Irrthum auf, wurde aber durch die Nierenverletzung und Störung der Circulation in der Niere verhängnissvoll für den Patienten. Die Anurie, wolcher der Patient erlag, erweckte während der klinischen Beobachtung die Meinung, dass die während der Operation unterbundene Arterie die Hauptarterie der Niere gewesen sei, und dass deshalb die Harnsecretion aufgehört habe. Es zeigte sich boi der Section, dass noch eine zweite, mindestens ebenso bedeutende Arterie die Niere mit Blut versorgte, so dass also das Stocken der Blutzufuhr nicht die Ursache der Anurie gewesen sein konnte. Man wird annehmen müssen, dass die Anurie reflectorisch entstanden sei, hat man doch bei Nephrectomie gelegentlich Anurie der gesunden, gänzlich unverletzten Niere eintreten sehen und als reflectorische Anurie bezeichnet. In dem vorliegenden Fall kann die beträchtliche Circulationsstörung, die in der Niere geschaffen warde, und die Verletzung des Nierenbeckens sehr wohl die Ursache für die Anarie gewesen sei.

\section{Fall IV.}

Gekreuzte Dystopie der rechten Niere und Verwachsung derselben mit dem unteren Pol der linken.

N...... Sch..., 79 Jahre, aus Rohrbacb, gestorben 9. April 1891.

Als zufälliger Sectionsbefund bei dem an Drüsencarcinom des Halses gestorbenen Manne wurde Folgendes gesehen:

Die beiden Nebennieren liegen an normaler Stelle und werden von normal verlaufenden Gefässen versorgt. Die linke Niere liegt an der gowöhnlichen Stelle; an ihrem unteren Pol ist sie mit der rechten Niere verschmolzen, welche völlig auf die linke Seite verlagert ist. Sie liegt in der Fossa iliaca sinistra, reicht in der Höhe der Aortentheilung bis in die Nittellinie und erstreckt sich nach abwarts bis zum Rand des kleinen Beckens. Mit ihrer unteren Hälfte bedeckt sie die Vasa iliaca sinistra. 
Die linke Niere ist von normaler Grösse und Gestalt. Ihr Hilus liegt an der inneren Seite des Organs und greift etwas auf die Vorderfläche über. Der linke Ureter verläuft über die Vorderfläche der verschmolzenen Organe herab in einer Furche, die der Grenze der beiden Nieren entspricht. Das ganze Organ ist $17 \mathrm{~cm} \mathrm{lang,} \mathrm{davon} \mathrm{entfallen} \mathrm{auf} \mathrm{die} \mathrm{linke} \mathrm{Niere} 10 \mathrm{cr}$. Die rechte Niere ist kleiner, als der Norm entspricht, $6 \mathrm{~cm}$ lang, $5 \mathrm{~cm}$ breit. Sie stellt ein rundes, plattes Gebilde dar, das an der Vorderfläche den Hilus trägt. Die Hinterfläche beider Nieren ist glatt. Ein eigentliches Nierenbecken ist an keiner der beiden Nieren entwickelt, sondern es kommen aus der Niere am Hilus eine Anzahl von Kanälen hervor, die sicb ohne Bildung eines grösseren Reservoirs direct zum Ureter vereinigen. Links sind 5 solcher Kanäle, rechts 6 entwickelt.

Der linke Ureter verläuft, wie erwähnt, in der Grenzfurche zwischen beiden Nieren, wendet sich dann an der linken Beckenwand herab zum Blasengrund, wo er einmündet. Der rechte Ureter kreuzt das Os sacrum und zieht vor den grossen Gefässen vorbei, an der rechten Beckenwand zum Blasengrund herab. Die Blase zeigt normale Verbältnisse, eben so die Geschlechtsorgane.

Die Gefässe, welche die Niere versorgen, sind folgende: Die Arteria renalis sinistra kommt in normaler Höhe aus der Aorta und tritt in 3 Aeste getheilt am Milus in die linke Niere ein. Die rechte Niere empfängt zwei Arterien: die Arteria renalis dextra superior geht zwei Finger breit oberhalb der Bifurcation aus der Aorta bervor, theilt sich in zwei Aeste, die an der Vorderfläche in die Niere eindringen, einer in den Hilus, einer direct in's Parenchym.

Eine Fingerbreite tiefer entspringt eine $\mathrm{zweite}$ Arterie aus der Aorta; sie verläuft an der Hinterfläche der rechten Niere berab, șchlägt sich um den unteren Rand derselben auf die Vorderfäche, um bier in den Hilus einzudringen.

Die Vena renalis sinistra sammelt sich aus mehreren Aesten, von denen einer aus der Vorderfläche der rechten Niere hervorgeht, und mündet an der gewöhnlichen Stelle in die Vena cava ascendens. Die beiden Venae renales dextrae laufen in Begleitung der Arterien und münden dicht oberhalb des Zusammenflusses der Venae iliacae in die Vena cava.

Der Fall giebt ein Beispiel für die als gekreuzte Dystopie bezeichneten Lageanomalien der Niere. Die rechte Niere ist völlig in die linke Körperhälfte verlagert und mit der normal gelegenen linken verwachsen. Ihre ursprüngliche Zugehörigkeit zur rechten Körperhälfte beweist der Verlauf und die Einmündung des Ureters in die rechte Blasenhälfte. Bemerkenswerth ist an dem Fall, dass ausser der Lageanomalie auch eine entschiedene Hypoplasie der rechten Niere besteht, bemerkenswerth ist auch das Verhalten des Nierenbeckens, das an beiden Nieren kein 
oinheitliches, sondern ein vielverzweigtes Gebilde ist. Erscheinungen während des Lebens hatte die Lageanomalie nicht gemacht.

Die hier beschriebenen Fälle geben Beispiele für die wichtigsten Typen ab, die man bei der Classification der Lageanomalien der Niere aufstellen kann. Bei der Eintheilung derselben kann man zunächst zwei grosse Reihen unterscheiden. In die erste Reihe rechnen alle Fälle, in denen sich nur eine Niere verlagert findet, die andere liegt dann entweder am normalen Ort oder sie fehlt gänzlich. In der zweiten Reihe von Fällen liegen beide Nieren am falschen Ort und zwar entweder getrennt oder in verschiedenen Graden mit einander verwachsen. Am häufigsten kommt unstreitig der Typus zur Beobachtung, den unser erster Fall demonstrirt: eine Niere liegt tief, die andere an normaler Stelle. Die linke Niere ist dabei häufiger verlagert als die rechte, eine Thatsache, die sich statistisch erbringen lüsst, ohne dass sich Gründe für die Verschiedenheit im Verhalten der rechten und linken Niere anführen liessen. Gruber hat 1866 einige Fälle von Tieflage der linken Niere beschrieben und im Aaschluss daran sämmtliche Fälle dieser Art aus der älteren Literatur reproducirt und auf's Eingehendste vergleichend erörtert. Den 36 von ihm besprochenen Fällen kann ich noch 11 in den letzten drei Jahrzehnten veröffentlichte hinzufügen, so dass mir mit dem von mir dargestellten Fall Beschreibungen. von 48 Fällen dieser Art vorliegen.

Ihnen stehen gegenüber 16 Beobachtungen von Tieflage der rechten Niere bei normaler Lagerung der linken.

Fall 2 und 3 unserer Beobachtung sind Beispiele für einen ungleich selteneren Typus der Nierendystopie, bei dem sich Tieflage einer Niere mit völligem Mangel der anderen vereinigt zeigt. Während der einseitige Nierendefect bei normaler Lage der anderen Niere, wie schon Eingangs erwähnt wurde, ein sehr gewöhnliches Vorkommniss darstellt, habe ich ausser meinen beiden Fällen, nur noch zwei weitere Fälle von Tieflage der einzigen Niere aufgefunden, die von Weisbach und Watson beschrieben worden sind.

Unter den Typus der gekreuzten Dystopie der Niere rechnen diejenigen Fälle, in denen die Niere einer Seite in die entgegen- 
gesetzte Körperhälfte unter die normal liegende verlagert ist, und sich entweder frei unter derselben vorfindet oder mit ihr verwachsen ist. Aus dem Verlauf des Ureters erkennt man in solchen Fällen, auf welche Körperhälfte die Niere ursprünglich gehört, denn bei nach links verlagerter rechter Niere mündet der Ureter in die rechte Blasenhälfte ein, und umgekehrt. Die Fälle von gekreuzter Dystopie ohne Verwachsung sind äusserst selten, nur Weisbach beschreibt einen Fall dieser Art. Die gekreuzte Dystopie mit Verwachsung, für die unser vierter Fall ein Beispiel liefert, ist häufiger. Meist ist dabei das obere Ende der verlagerten Niere mit dem unteren Pol der normal gelegenen verwachsen. Nicht selten ist dann aber auch die obere Niere nicht ganz normal gelagert, so dass sich Uebergangsformen von dieser Anomalie zu dem gleich zu besprechenden Typus, der Dystopie beider Nieren mit Verwachsung, zeigen.

In die oben aufgestellte zweite Reihe von Fällen gehören diejenigen Beobachtungen, bei denen sich beide Nieren verlagert erweisen. In dieser Reihe unterscheidet man wieder zwei Typen; entweder sind beide Nieren unabhängig von einander, oder, der häufigere Fall, sie sind in geringerem oder höherem Grade mit einander verwachsen. Verlagerung beider Nieren ohne Verwachsung habe ich nur in vier Fällen beschrieben gefunden, und zwar von Martin St. Ange, Boyd, Potherat und Poirier. Im Fall von Martin St. Ange lagen beide Nieren eines, bald nach der Geburt gestorbenen, Kindes im kleinen Becken, ohne mit einander verwachsen zu sein. Boyd beschreibt einen Fall mit Tieflage beider Nieren in den Fossae iliacae, ebenso Potherat, bei Poirier fand sich eine Niere im kleinen Becken, die andere in der Darmbeingrube.

Diesen sehr seltenen Formen gegenüber, zählt der Typus der Dystopie beider Nieren mit Verwachsung zu den häufigeren Anomalien. Es hat, wie schon erwähnt, dieser Typus Beziehungen zu den Formen der gekreuzten Dystopie mit Verwachsung. Stocquart hat 1878 einen Fall dieser Art beschrieben und viele Fälle aus der älteren Literatur zusammengestellt. Ich habe im Ganzen 33 Fälle von gekreuzter Dystopie mit Verwachsung und Dystopie beider Nieren mit Verwachsung aufgefunden. Man kann die letzteren Formen wieder sondern 
in dystope Tufeisennieren und dystope Kuchennieren. Bei der Hufeisenniere sind nur die unteren Pole mit einander verwachsen. Fast jede Hufeisenniere zeigt einen geringen Grad von Verlagerung ihrer beiden Componenten nach unten. Nur die höheren Grade der Dystopie habe ich jedoch in meiner Zusammenstellung berücksichtigt.

Von diesen Formen findet ein continuirlicher Uebergang statt, zu den als dystopen Kuchennieren bezeichneten, indem sich bei stärkerer Verlagerung der beiden Nieren auch leicht die typische Hufeisenform verliert und an ihrer Stelle ein unregelmässig verwachsenes, scheinbar einheitliches Organ entsteht, das von den älteren Beobachtern auch vielfach für eine einzige Niere gehalten worden ist. Das Vorhandensein von zwei Ureteren, die in je eine Blasenhälfte einmünden, ist in solchen Fällen das entscheidende Kriterium dafür, dass man das Organ als Verschmelzungsprodukt zweier ursprünglich getrennter Nierenanlagen anzusprechen hat.

Zum Scbluss seien der Vollständigkeit halber noch die seltenen Fälle von mehrfacher Nierenentwickelung auf einer Seite erwähnt, die dann unter einander za liegen pflegen. Blasius giebt die Beschreibung und Abbildung eines Falles, bei dem sich rechts zwei unter einander liegende Nieren finden, deren Ureteren beide in die rechte Blasenhälfte einmünden. Die linke Niere liegt an normaler Stelle. Ein analoger Fall ist in Schmidt's Jahrbüchern ohne Namenangabe des Beobachters erwähnt. Aus früherer Zeit existiren eine Reihe von Angaben über 3fache, 4fache, selbst 5fache Nieren, die Fälle sind jedoch nicht näher beschrieben und es handelt sich dabei wahrscheinlich meist um verwachsene Nieren, die mit ihren Buckeln und vielverzweigten Nierenbecken fälschlich für das Conglomerat einer Anzahl von Nieren gehalten worden sind. Kriterium ist auch in diesen Fällen die Anzahl und Einmündungsstelle der Ureteren.

Man findet bei Lageanomalien der Nieren regelmässig in der Form, Grösse, Gefässversorgung, in der Lage der Hilus des dystopen Organs, ferner in der Länge und dem Verlauf des Ureters Abweichungen von der Norm. Man findet auch nicht selten an den benachbarten Organen Situsveränderungen und 
sonstige Anomalien, die theils durch die Verlagerung der Niere hervorgerufen sind, theils zulälig mit derselben coincidiren. Diese auatomischen Verhältnisse sind etwas eingebender zu besprechen, vorher sei in Kürze die Frage nach dem Wesen und der Entstehung der Nierendystopie erörtert.

Unter Berücksichtigung des frühesten Auftretens der Nierenanlage im fötalen Organismus, sowie der Situsveränderung, welche die embryonale Niere eingeht, sind wir berechtigt, die Tieflage der Niere als eine Entwickelungshemmung aufzufassen. Es handelt sich in allen Fällen von angeborner Tieflage um eine primäre Situsanomalie, nicht um ein späteres Herabrücken der Niere. Die Entwickelungsgeschichte lehrt uns Thatsachen kennen, aus denen sich die Möglichkeit eines Zustandekommens der Tieflage ergiebt, über die Ursachen der Hemmungsbildung giebt uns die Entwickelungsgeschichte dagegen keine Anhaltspunkte. Diese Ursachen bleiben uns daher überbaupt verschlossen, denn auch aus den anatomischen Verhältnissen, die wir im fertigen Organismus bei Nierentieflage finden, sind sie nicht zu ergründen.

Die Niere geht aus einer Ausstülpung des Urnierengangs hervor, der in eine am hinteren Ende der Urniere gelegene Wucherung der Mittelplatte hineinwächst und mit dieser die erste Anlage der bleibenden Niere darstellt. Die Anlage kommt jedoch nicht am Ort ihres ersten Auftretens zur definitiven Entwickelung, sondern sie wächst hinter der Urniere empor und bildet sich erst am oberen Ende des Wolff'schen Körpers zu ihrer späteren Grösse und Struktur aus. Dabei kommt es zugleich zu einer Drehung des Unnierengangs um $180^{\circ}$, wodurch der ursprünglich hinter ihm liegende Ureter vor ihn gelangt. Indem das gemeinschaftliche Stück des Urnierengangs und Ureters allmählich in den Sinus urogenitalis hinein bezogen wird, verliert der Ureter seine directe Beziehung zum Urnierengang. Ihre Mündungen rücken immer weiter aus einander, der Ureter mündet jetzt in die Allantois, die später zur Harnblase wird. Die beiden Nierenanlagen liegen im frühesten Stadium ihrer Entwickelung ganz nahe der Mittellinie in der Gegend des 31. bis 34. Ursegments.

Wie Kupfer bei einem Schafembryo von $10 \mathrm{~mm}$ Länge beschreibt, sind beide Nieren in dieser Entwickelungsstufe von 
Past sphärischer Form, berühren sich in der Mittellinie, liegen hart vor der Theilungsstelle der Aorta und nehmen fast den ganzen Raum zwischen dieser und der Wurzel des Mesenteriums ein". In späterer Zeit fand er die Nieren weiter nach vorn gerückt und die Aorta zwischen ihnen.

Aus diesen entwickelungsgeschichtlichen Thatsachen folgt, dass die Tieflage der Niere auf einem Ausbleiben des Wachsthums längs des Wolf'schen Körpers berwht. Mag ein mechaniches. Moment oder mangelnde Wachsthumsenergie die Entwickelungsstörung verursachen, jedenfalls erreicht die Niere nicht das Endziel ihrer Wanderung, sondern bleibt an irgend einer Stelle der Wegstrecke, die sie embryonal zurïcklegen soll, liegen und kommt daselbst zu ihrer definitiven Ausbildung. - Man kann nach diesen Auseinandersetzungen sich leicht die verschiedenen Grade der Dislocation, vom kleinen Becken bis dicht unterhalb der normalen Stelle, sowie auch die Verschiedenheit der Lage zur Mittellinie des Körpers ableiten. Auch die Möglichkeit einer Verwachsung beider Nieren ergiebt sich aus der Berührung der Nierenanlagen in den ersten Stadien, und, je nachdem dann die eine oder andere Niere oder beide ihre Wanderung längs der Uniere fortsetzen, je nach dem Grad der Verschmelzung ferner, kommt es zur rechts oder linksseitigen Dystopie der verwachsenen Niere oder zur normal gelagerten Hufeisenniere.

Eine Reminiscenz an embryonale Verbältnisse erhält sich bei der tiefliegenden Niere meist in der Form des Organs und der Lage des Hilus. Die Form erinnert an die sphärische Gestalt der Nierenanlage, von der Kupfer berichtet. Die Niere stellt in der Regel ein ovales oder rundliches Gebilde dar, das, von vorn nach hinten abgeplattet, an der Vorderfäche den Hilus trägt, aus dem das Nierenbecken hervorgeht, und in den die Hauptarterien eindringen. In den vier Fällen unserer Beobachtung fand sich der Hilus stets an der Vorderfiäche, und so ist es fast ausnabmslos der Fall. Von seltenen Ausnahmen berichten Eustachi, Durham und Gruber. Sie fanden den Hilus an der Hinterfiäche des Organs entwickelt.

Eine Beeinflussung erfährt die Form der Niere durch die Räumlichkeit, der sie sich anzupassen hat, und durch die umgebenden 


\section{7}

Weichtheile. So ist ganz charakteristisch der durch den Psoas hervorgerufene Eindruck unseres dritten Falles. Auch Weisbach und Stern haben einen solchen Eindruck beobachtet; Goux berichtet, dass die Niere durch die Aorta, Schott, dass sie durch die Arteria iliaca einen Eindruck an ihrer hinteren Fläche erhalten habe. Liegt die Niere zum Theil im kleinen Becken, so hat sie an ihrer Hinterfläche eine Furche, entsprechend dem Eindruck, den der Rand des kleinen Beckens gemacht hat, sie ist gleichsam über die Fläche gebogen. Liegt die Niere ganz im kleinen Becken, so passt sie sich völlig den Raumverhältnissen desselben an, sie zeigt dann an Stelle der plattovalen, eine mehr viereckige Form. Sehr schön ausgeprägt findet sich dies Verhalten im zweiten unserer Fälle, wo die Niere bei einem Neugeboruen die ganze Cavität des kleinen Beckens ausfüllt. Thre Hinterfläche ist in solchen Fällen convex gestaltet, in die Concavität des Kreuzbeins eingefügt, während der Hilus aus der concaven Vorderfläche hervorgeht. Das ganze Organ verschmälert sich nach unten.

Die verwachsene Niere zeigt je nach dem Grad der Verschmelzung der beiden Organe verschiedene Form und Bildung. Die typische Hufeisenform findet sich namentlich in denjenigen Fällen, wo die Tieflage keine sehr beträchtliche ist. In anderen Beobachtungen stellen die verwachsenen Organe einen platten langgestreckten Körper dar, dessen Längsaxe in die Längsaxe des Körpers fällt oder quer oder schief zu derselben orientirt ist. So giebt Monginot die Beschreibung und Abbildung eines Falles, bei dem die verwachsenen Nieren in der Lumbalgegend quer über die Wirbelsäule ausgebreitet waren, lang gestreckt, aus mehreren von einander abgrenzbaren Lappen zusammengesetzt. Es gingen 3 Ureteren aus dem Organ hervor, über deren weiteren Verlauf jedoch nichts ausgesagt wird. Von Anderen wurde der oblonge oder S-förmig gekrümmte Nierenkörper längs liegend ganz in einer Körperhälfte angetroffen, wie es ähnlich ja auch in einem unserer Fälle statt hat. Endlich können verwachsene Nieren zu einem rundlichen, platten Gebilde gestaltet sein, das aus unregelmässigen Knoten und Lappen besteht. Man bezeichnet diese Form als Kuchenniere, von der unter Anderen Haller ein sehr schönes Beispiel beschrieben 
und abgebidet hat. Der Hilus liegt auch bei den verwachsenen Nieren in der Regel vorn and die Ureteren verlanfen über die Vorderfläche der Niere herab, der Blase zu.

In der Form und Bildung des Nierenbeckens zeigen sich bei den Lageanomalien der Niere recht häufig ungewöhnliche Befunde. Wie dasselbe in allen unseren Fallen aus mehreren Aesten zusammenfloss oder auch gar nicht entwickelt war, so dass die Nierenkelche sich direct in einen Ureter vereinigten, so wurden in einer grossen Anzahl der Beobachtungen Varietäten dos Nierenbeckens gefunden. Dagegen ist das Restiren der embryonalen Lappung wohl hin und wieder beobachtet worden, seheint mir aber bei der tiefliegenden Niere nicht häufiger vorzukommen, als auch sonst. In unseren Fällen bestand der Lappenbau der Niere nicht. Die Furchen, die sich sehr regelmässig an der Vorderfäche der tiefliegenden Niere finden, entsprechen den auch bei normaler Niere in der Nähe des Hilus auftretenden Furchen.

Die Grösse der verlagerten Niere ist in den einzelnen Beobachtungen sehr verschieden gefunden worden. Das gewöhnliche Verhalten ist, dass die dystope Niere der normal gelagerten an Grösse gleich kommt. Eine bemerkenswerthe Abweichung von diesem Verhalten weisen diejenigen Fälle auf, in denen die tiefliegende Niere hypoplastisch ist. Man wird nicht fehl gehen, wem man in diesen Fällen die congenitale Atrophie oder Hypoplasie mit der Tieflage auf dieselbe Ursache zurückführt; es handelt sich dann um eine Entwickelungshemmung, die sowohl in der Grösse und Struktur des Organs als auch in seiner Lage zum Ausdruck kommt. In geringem Grade trat ein Zurückbleiben in der Grösse des verlagerten Organs in unserem vierten Fall in die Erscheinung, wo das Durchschnittsmaass der Grösse bei weitem nicht erreicht wurde. Ausgeprägte Fälle von Нypoplasie haben Gruber ${ }^{1}$ ), Westphal ${ }^{2}$ ), Chambonde Montaux ${ }^{3}$ ) beobachtet und beschrieben. Der Gruber'sche Fall zeigte ausserdem eine interessante Missbildung des Ureters, und sei deshalb in Kürze wiedergegeben.

1) Dieses Archiv. 1876.

2) Berl. klin. Wochenschr. 1890.

3) Observ. clinic. Paris 1789. 
Der Ureter, ein sehr stark erweiterter kurzer Kanal, mündete nicht in die Harnblase, sondern endigte blind und hatte nur seitlich eine Communication mit der Vesica seminalis. Die Folge dieser Missbildung war gewesen, dass die Niere, deren Secret keinen Abfluss hatte, in ihrer Entwickelung zurückblieb und atrophirte. Sie stellte eine in der Höhe des vierten Lendenwirbels liegende, kleine Cyste dar, ohne Spur von Drüsensubstanz. Die normal liegende linke Niere war compensatorisch hypertrophirt. Gruber deutet den Fall so, dass der Ureter nie die normalen Communication mit der Blase erlangt habe, und dadurch Anlass zu der Atrophie der Niere gegeben habe. In dem von Westphal beschriebenen Fall lag die congenital atrophische Niere tief in der Fossa iliaca. Chambon de Montaux erzählt von einer Niere, die er dem Uterus aufsitzend fand, bedeutend kleiner als der Norm entspricht.

Im Gegensatz zu diesen Fällen von abnormer Kleinheit des tiefliegenden Organs, wies unser dritter Fall eine dystope Niere von ungewöhnlicher Grösse auf. Da es sich dabei gleichzeitig um Fehlen der anderseitigen Niere handelte, ist dies Hypervolum durch die vermehrten Ansprüche zu erklären, welche an die Function des Organs gestellt wurden. Solche compensatorische Hypertrophie ist bei Fällen von congenitalem Nierenmangel ein sehr gewöhnliches Vorkommniss und in der Literatur des Nierendefectes ${ }^{1}$ ) findet man die Frage der Hypertrophie und der mikroskopischen Befunde bei derselben auf's Eingehendste besprochen. Der Ureter ist in allen Fällen von Nierendystopie, entsprechend dem Grad der Tieflage, verkürzt, häufig etwas weiter als normal. Sein Verlauf ist häufig geschlängelt. In seiner Einmündung in die Blase zeigt er dagegen fast stets normales Verhalten. Das ist ja unschwer verständlich, wenn man bedenkt, dass die Entwickelung der Niere mit einer Wucherung des Ureters beginnt, die Bildung and Einmündung desselben also von der sich später ausbildenden Tieflage der Niere in keiner Weise beeinflusst werden kann. Eine Missbildung des Ureters, wie sie der eben beschriebenem Fall Gruber's zeigt, stellt eine seltene Ausnahme dar.

${ }^{1)}$ Beumer, dieses Archiv. Bd. 72. - Guttmann, ebendaselbst. Bd. 92.

- Eckardt, ebendaselbst. Bd. 114. 
Ein grosser Reichthum von Varietäten tritt in der Blutversorgung der verlagerten Niere zu Tage. Es kommen nach Zahl, Ursprung und Verlauf der Gefässe Schwankungen innerhalb so weiter Grenzen vor, dass jeder einzelne Fall in dieser Hinsicht seine Besonderheiten hat. Allen gemeinsam ist, dass die Gefässe dor tiefliegenden Niere tieferen Ursprangs sind, als die aormale Arteria renalis, und dass die Zahl der Gefässe gegenüber der Norm vermehrt ist. Kommt schon bei der normal gelagerten Niere nicht allzu selten eine Vermohrung in der Zahl der Arterion vor, wofür der zweite Fall unserer Beobachtung ein Beispiel liefert, so ist eine Mehrzahl der Arterien die ausnahmslose Regel bei der verlagerten Niere. Es wurden bis zu כ Arterien beobachtet, 2-3 sind der gewöhnliche Befund. Diese entstammen entweder den tieferen Abschnitten der Aorta nahe der Bifurcation, oder sie gehen aus einer der Hiacae hervor. Häufig wird die Niere aus mehreren Quellgebieten versorgt, nicht selten bezieht sie ihr Blut aus Arterienstämmen beider Körperhälften.

Wenn man sich klar macht, dass die Niere bei ihrer embryonalen Wanderung das Gebiet verschiedener Arterien passirt, und dass die Tieflagerung der Niere nichts als die Fixation irgend eines dieser embryonalen Stadien bedeutet, so lässt sich der Wechsel in dem Gefässbefund leicht verstehen. So lange die Nierenanlage vor dem Promontorium liegt, gehört sie in's Gebiet der Hypogastricae. Sie rückt dann weiter nach vorn; in der Fossa iliaca wetteifern Iliaca communis und Aorta an die Versorgung des Organs mit Blut; gelangt sie endlich an den Platz ihrer Bestimmung, so füllt allein der Aorta die Aufgabe der Ernährung za, und es kommt zur Ausbildung der definitiven Gefässbahnen zur Niere. Bleibt die Niere an irgend einer Stelle ihres Weges liegen, so wird sie den Arterienstämmen tributär, in deren Bereich sie liegt. Die bedentendsten Abweichungen von der Norm finden sich daher bei Tieflage im Slleinen Becken, wo die Aorta and die Lliacae beider Körperhälften an der Versorgung des Organs Antheil suchen. In solchen Fällen - Fall 1 unserer Beobachtung gehört dazu - findet man nicht nur die grösste Zahl von Arterien, sondern auch die grösste Verschiedenheit in Ursprungsgebiet derselben. Rückt 
die Niere längs der Wirbelsäule auf einer Seite in die Höhe, so beschränkt sich die Blutzufuhr zunächst auf eine Körperhälfte, ferner nimmt die Zahl der Arterien mehr und mehr ab, und findet man die Niere nur wenig unterhalb ihrer normalen Stelle gelagert, so zeigt sie, wie in der Form und in der lage des Hilus, so auch in der Gefässversorgung, Verhältnisse, die der Norm sehr nahe stehen.

Für den Eintritt der Gefässe ist zwar meistens der Hilus der bevorzugte Ort, doch kommen auch mannichfach andere Befunde zur Beobachtung, indem die Arterien direct in die Substanz der Nieren eindringen, sei es in der Nähe des Hilas, sei es an der Hinterfläche der Niere oder an einem der Pole. Unsere Fälle geben für alle diese Verhältnisse gute Beispiele, so dass es nicht nöthig ist, auf andere Beobachtungen zu exemplificiren.

Fast das gleiche Verhalten, wie die Arterien, zeigen die Venen. Ihre Zahl kann auch bis auf fünf und mehr steigen, häufig jedoch fliessen die Venenäste zu einem oder zwei Hauptästen zusammen, die sich in die Vena cava ascendens oder die Vena iliaca communis ergiessen. Die Venen verlaufen in Begleitung der Arterien oder treten an beliebiger Stelle aus der Substanz der Niere hervor.

Pathologische Zustände trifft man an der tiefliegenden Niere nicht häufiger oder seltener, als an der normal gelagerten. Es kommen gelegentlich die verschiedenen Formen der Nephritis (Weisbach) und Hydronephrosenbildung (Gruber) vor. Kruse berichtet von einem Fall von partieller Hydronephrose einer verschmolzenen Niere; Drouin von einer vor dem Sacrum liegenden Niere, welche acht Steine bis zu Taubengrösse enthalten habe. Kaltschmidt erzählt von einer alten Frau, die ohne vorhergegangene Nierensymptome, plötzlich mit heftigen Nierenschmerzen erkrankte und unter den Erscheinungen der Anurie nach wenigen Tagen starb. Man fand bei der Section die beiden Nieren verschmolzen, tiefliegend und das Nierenbecken mit Steinen erfüllt.

Einen besonders hohen Procentsatz von Erkrankungen liefern die dystopen Nieren jedoch nicht. Dass Leute, die einen congenitalen Nierendefect oder eine verschmolzene Niere besitzen, bei pathologischen Vorgängen in diesem Organ, einem besonders 
grossen Risiko ansgesetat sind, ist ohne Weiteres einlenchtend und wird auch durch den dritten Fall unserer Beobachtung demonstrirt.

Den von der Norm abweichenden anatomischen Verhältnissen, die an der Niere selbst, bei Dystopie des Organs, hervortreten, gesellen sich eine Reihe von Lage- and Formanomalien der boanchbarten Abdominalorgane hinzu.

Ein Befund, auf den schon die älteren Beobachter von Lageanomalien der Nieren aufmerksam wurden, und der mit grosser Regelmässigkeit erhoben worden ist, ist der, dass die Nebenwiere ihren normalen Platz inne hat, wenn die Niere dislocirt ist. Die Zusammengehörigkeit der Glandula suprarenalis mit der Niere ist ja seit Langem als eine rein topographische erkannt worden, die Entwickelungsgeschichte lehrt die Cnabhängigkeit beider Organe von einander, and auch diese regelmässige Wahrnehmung des pathologischen Anatomen weist auf diese Unabhängigkeit hin. Die Entwickelungsgeschichte lehrt bekanntLich, dass die Nebennieren zum Theil von den Ganglienanlagen des sympathischen Grenzstrangs abstammen, zum Theil aus einer Zellanbäufung hervorgeht, bei deren Bildung die vordersten Theile der Urniere betheiligt zu sein scheinen. Jedenfalls ist also die Anlage der Nebenniere von der ersten Nierenanlage, sowobl dem Entstehungsboden, als dem Ort der Entstehung nach, völlig getrennt. Niemals liegt daher die Nebenniere dem oberen Pol der tiefliegenden Niere auf, dagegen findet man häufig auf der Seite, wo die Niere tiefliegt, auch einen geringen Grad von Tieflage der Nebenniere. Dieselbe ist wohl einfach mechanisch zu erklären, indem der Druck, den die Niere normaler Weise gegen die Nebenniere ausübt, hier weggefallen ist. Ausserdem kommt gelegentlich auch bei normaler Lage der Niere Tieflage der Nebenniere vor ${ }^{1}$ ). Fälle von Mangel einer oder der beiden Nebennieren sind, wenn sie mit Tieflage der Niere gleichzeitig beobachtet werden, als zufälliges Zusammentreffen zu deuten ${ }^{2}$ ).

Die tiefliegende Niere wird, wie die normal gelagerte, an ihrer Vorderfläche vom Peritonäum überkleidet, bei Tieflage im

1) Fried lowsky, Allg. Wiener med. Ztg. 1867.

9) Fälle von Scheiber, Wien. med. Jahrb. 1875. - Stocquard, a. a. O. - Pacoud, l. c. 
kleinen Becken findet sich nur ein Theil der Vorderfläche mit einem serösen Ueberzug bedeckt. Das Peritonäum schlägt sich dann von der Niere auf die Blase oder das Rectum über, eine Excavatio vesico-renalis oder recto-renalis bildend, wie sie sich im 2. und 3. Fall unserer Beobachtung ausgebildet finden und auch von Gruber u. A. beschrieben worden sind.

Die Blase wird durch die tiefliegende Niere in ihrer Gestalt und Lage nicht beeinflusst. Die Asymmetrie des äusseren und inneren Baues der Blase, wie ihn zwei unserer Fälle zur Anschauung bringen, ist der gewöhnliche Befund, der bei einseitigem Nierendefect erhoben wird. Im 3. Fall waren durch das Bestehen einer Phimose und der Atresia ani vesicalis besondere Bedingungen geschaffen, auf welche die ungewöhnliche Ausdehnung des Organs zu beziehen ist.

$Z_{\text {wei }}$ unserer Fälle boten Anomalien der Venenverzweigung des Beckens dar, indem einmal die linke Vena hypogastrica in die rechte Vena iliaca communis mündete, das andere Mal das Blut der linken Hypogastrica zum Theil in die rechte Hypogastrica hinübergeleitet war, zum Theil normal in die Iliaca communis sinistra einmündete. Es ist merkwürdig, dass ähnliche Anomalien der Beckenvenen auch von Gruber ${ }^{1}$ ) in 4 Fällen von Tieflage der Niere beobachtet worden sind.

Der Darm weist bei dystoper Niere am häufigsten Lageabweichungen auf. Besonders die Tieflage der linken Niere im kleinen Becken oder nahe dem Beckeneingang ruft olt Situsanomalien des Darms hervor. In den 33 Fällen, die Gruber zusammengestellt hat, fand sich das Rectum 11 mal nach rechts verlagert, in den später veröffentlichten Fällen finde ich das gleiche Verhalten noch 3 mal erwähnt ${ }^{2}$ ). Auch Fehlen der Flexura sigmoidea, Verdoppelung und Verlagerung derselben sind beschrieben ${ }^{3}$ ). Alle diese Anomalien charakterisiren sich ohne Weiteres als Folgen der Tieflage der Niere. Indem die Niere sich an der Stelle einlagert, wo normaler Weise das S romanum oder der Anfangstheil des Rectum zur Entwickelung kommt,

1) Gruber, Wien. med. Jahrb. 1866. Dieses Archiv. Bd. 78.

2) Gruber, dieses Archiv. Bd. 78. - Friedlowsky, a. a. 0. Stern, a. a. 0 .

3) Gruber, Wien. Jabrb. 1866. 
veranlasst sie die abweichende Lagerung und Bildung des Darms. Ebenso verhält es sich mit einer Verlagerung des Uterus nach links in einem Fall, den Boinet ${ }^{1}$ ) beschreibt. Die Niere lag hier im kleinen Becken und zwar hinter der Blase; der Uterus war nach links und hinten gedrängt, das Ligamentum latum der rechten Seite $z w i s c h e n$ Niere und Rectum ausgespannt.

Eine ganz andere, ungleich seltenere Bildungsanomalie des Darms demonstrirt der zweite unserer Fälle, wo sich eine tief im Becken liegende Niere gleichzeitig mit Atresia ani vesicalis vorfand. Die Wahmehmung ist von ganz besonderem Interesse. Nicht als ob nicht schon gelegentlich Atresia ani und Dystopie der Niere zusammen vorgekommen wären, sondern weil man in diesem Fall einen causalen Zusammenhang zwischen den beiden Missbildungen wohl annehmen darf. Beim Vergleich mit den in der Literatur beschriebenen Fällen ähnlicher Art wird das Charaliteristische unseres Falles hervortreten. Bousquet ${ }^{2}$ ) fand bei einem bald nach der Geburt gestorbenen Mädchen die rechte Niere tief, auf dem 4. Lendenwirbel, liegend. Die Blase fehlte. Die beiden Ureteren mündeten, nach seiner Beschreibung, zwischen den Schamlippen. Auch ein After war nicht vorhanden. Der Enddarm mündete in die Scheide. Godard ${ }^{2}$ ) sah eine in der linken Darmbeinschaufel liegende verschmolzene Niere und fand gleichzeitig Offensein des Urachus und Atresia recti. Ausser mit Dystopie der Niere hat man gelegentlich mit anderen Bildungsfehlern der Niere Atresia ani combinirt gefunden. Ich erwähne hier eine Beobachtung von Scheiber ${ }^{3}$ ), der bei einem 30jährigen Mädchen Atresia ani vaginalis beobachtete und gleichzeitig Fehlen der rechten Niere und Nebenniere vorfaud. Auch unser Fall war ausser durch Tieflage einer Niere durch Mangel der anderen ausgezeichnet. Gerster ${ }^{4}$ ) hat Zusammentreffen von Hufeisenniere und Atresia ani beschrieben. In seinem Fall hatte der linke Ureter den normalen Anschluss an die Blase nicht erreicht, sondern mündete in das blind endigende Rectum. Durch Stauung des Harns in diesem Blindsack kam es zur

) Boinet, siehe Literaturverzeichniss.

2) Literaturangaben am Schluss.

3) Wien. med. Jahrb. 1875.

†) New York med. Journal. 1878. 
Hydronephrosenbildung schon während des Fötallebens. Der Tod trat bald nach der Geburt ein und die Section deckte diese seltene Missbildung auf.

Alle diese Thatsachen beanspruchen Interesse, insofern sie eine Combination verschiedener, nicht sehr gewöhnlicher Missbildungen darstellen, und damit zugleich einen Beleg für die Erscheinung liefern, dass sich oft eine Neigung zur Häufung von Missbildungen beobachten lässt. Unser Fall nimmt eine Sonderstellung ein, insofern es sich bei ihm nicht nur um ein Nebeneinander verschiedener Bildungsfehler handelt, sondern ein cau- saler Zusammenhang zwischen beiden zu erkennen ist. Wenn Eppinger ${ }^{1}$ ), nach Analogie mit Hemmungsbildungen anderer Organe, alle Fälle von Atresia ani auf mechanische ätiologische Momente zurückführen möchte und dafür einige Belege erbringt, so dürfte unser Fall eine wichtige Stütze seiner Ansicht liefern. Denn es ist unschwer ersichtlich, dass die völlig in's kleine Becken gerückte Niere, welche dem Beckenboden and Damm fest auflag, nicht nur den Enddarm verlagern, sondern auch der Ausbildung eines Anus ein mechanisches Hinderniss bieten musste. Es erlangte somit nur der vordere Theil der Cloake eine Oeffnung nach aussen, nur die Harn- und Geschlechtsausführwege bildeten sich normal aus; der Endarm blieb von der Verbindung mit Körperoberfläche abgeschlossen, es persistirte eine Cloake, die in der Verbindung von Rectum und Blase zum Ausdruck kommt.

Noch häufiger als mit Bildungsfehlern des Intestinaltractus findet man die Dystopie der Niere mit Missbildungen der Geschlechtsorgane, besonders der Gescblechtsausführwege vereinigt. Auch für eine derartige Combination giebt ein Beispiel unser dritter Fall, bei dem wir Kryptorchismus und eine Anomalie der Geschlechtswege constatiren konnten, neben Tieflage einer und Fehlen der anderen Niere. In der Literatur sind mehrfach ähnliche Fälle beschrieben worden. Der retinirte und atrophische Hoden unseres Falles gehörte der Körperhälfte an, deren Niere gar nicht zur Entwickelung gekommen war, und wenn auch das Präparat eine genaue Feststellung der Verhältnisse des Vas deferens und der Samenblase nicht mehr zuliess, so erinnert

1) Prager med. Wochenschr. 1880. 
dooh der Fall lebhaft an die wiederholt gemachten Wahrnehmungen, bei denen sich gleichzeitig mit Nierendefect eine Agenesie der Geschlechtsausfuhrwege vorfand, und in Folge letzterer oine Atrophie des Hodens eingetreten war. (Gruber ${ }^{1}$ ), Mosler ${ }^{2}$ ), Bachhammer ${ }^{3}$ ) haben solche Fälle, die völlig mit einander übereinstimmen, vorgebracht. Letaterer erörtert im Anschluss an seine Beschreibung die Entstehung dieser Anomalie und bringt eine sehr plausible Ansicht über das Zustandekommen derselben vor. Er weist darauf hin, dass in diesen Fällen alle rom Urnierengang sich ableitenden Gebilde fehlen, Ureter und Niere, Vas deferens und Samenblase, und dass es sich daher wohl nicht um eine zufällige Coincidenz verschiedener Missbildungen handeln werde, sondern der Ursprung aller der gleiche sei, gelegen in einer Entwickelungsstörung, die den Urnierengang betroffen habe.

Es ist interessant, dass auch beim weiblichen Geschlecht ähnliche und ähnlich zu deutende Bildungsfehler beobachtot worden sind. Ich stelle vier Fälle zusammen, die ich aus der Literatur gesammelt habe, und deren Uebereinstimmung frappant ist. Luton ${ }^{4}$ ) sah eine tiefliegende linke Niere in der Leiche eines bald nach der Geburt gestorbenen weiblichen Kindes, bei dem gleichzeitig das linke Uterushorn rudimentär war, die linke Tube fehlte, und das linke Ovar an Grösse beträchtlich hinter dem rechten zurückblieb. Kussmaul ${ }^{5}$ ) hat die Beschreibung eines Präparats der Freiburger anatomischen Sammlung gemacht. Dasselbe stammte von einer 6jjährigen Frau und zeigte eine tiefliegende linke Niere; linkes Uterushorn und linke Tube waren als Rudimente kaum zo erkennen; das linke Ovarium war gleichfalls rudimentär. Rechts bestanden normale Verhältnisse.

Boyd ${ }^{6}$ ) giebt den Sectionsbefund einer 72jährigen Frau:

1) Gruber, Lémoires de ''Acad. de St. Petersbourg, VIL. série. Tome IL. 1860.

2) Mosler, Archiv für Heilkunde. 1863.

3) Bachhammer, Archiv für Anat. 1879.

†) Luton, Comptes rendus de la Société de biologie. 2. sér. Tome 4. 1857.

3) Kussmaul, Mangel, Verkürnmerung, Verdoppelung der Gebärmutter. Würzburg 1859.

o) Boyd, Medico-chir. transact. London 1841. 


\section{7}

beide Nieren liegen tief, das rechte Ovarium ist Sitz eines Tumors, das linke ist rudimentär. Beide Uterushälften, beide Tuben fehlen. Von der Vagina ist nur eine Andeutung vorhanden. Braxton Hicks') endlich berichtet, dass er bei einer Section die linke Niere nach rechts unten verlagert und mit der rechten verwachsen gefunden habe. Die linke Uterushälfte mit ihren Adnexen feblte.

Die vier Fälle sind so ausserordentlich übereinstimmend im anatomischen Befund, dass man schon dadurch auf eine den Bildungsfehlern der Niere und der Geschlechtsorgane gemeinschaftliche Grundursache hingewiesen wird. Es ist anzunehmen, dass auch hier die ursprüngliche Entwickelungsstörung den Urnierengang betroffen hat, von dem sich sowohl die Nierenanlage als die Müller'schen Gänge ableiten, welche später die weiblichen Geschlechtsausführwege, Tube, Uterus und Vagina, hervorgehen lassen. Eine Anzahl von Fällen, wo es sich um einseitigen Nierendefect mit Anomalien der weiblichen Genitalorgane bei normaler Lage der anderen Niere handelt, finden sich bei Beumer (a. a. 0.) zusammengestellt.

Die praktischen Erfahrungen, die man bisher über die Nierendystopie gesammelt hat, stehen an Reichhaltigkeit bei Weitem hinter den mannichfaltigen anatomischen Beobachtungen über diesen Bildungsfehler zurück. Und es ist anzunehmen, dass auch in Zukunft die Nierendystopie häufiger bei der Section, als in der Klinik zur Beobachtung kommen wird, denn die überwiegende Mehrzahl der Fälle machen keine klinischen Erscheinungen, verursachen dem Träger keine Beschwerden und dem Arzt kein Kopfzerbrechen.

Es existiren eine grosse Anzahl von Beschreibungen, bei denen die Lageanomalie der Niere zufällig in der Leiche gefunden wurde, nachdem der Besitzer sie 70 oder selbst 80 Jahre ohne Beschwerde mit sich herumgetragen hatte. Und in denjenigen Fällen, wo der Finger des Arztes die Niere als Tumor an der hinteren Bauchwand prominiren füblte, blieb es in früherer Zeit beim Staunen über diesen unerklärlichen Befund, für ein ärztliches Eingreifen hätte ein solcher Tumor keine Indication abgegeben, selbst wenn er bedrohliche Krankheits-

1) Braxton Hicks, Transact. of obstetric. Soc. London 1879. 
symptome hervorgerufen hatte, was meistens nicht der Fall war. Im letaten Punkt hat sich die Sache geändert, seitdem die Chirurgie vor einer Eröffnung der Bauchhöhle nicht mehr zurückzuscheuen brancht. Die Frage nach der praktischen Bedeutung der Nierendystopie ist in ein anderes Stadium getreten, seitdem die Eventualität der Operation eines auch seinem Wesen nach nicht genau zu diagnosticirenden Tumors in Betracht kommt, falls derselbe das Leben zu geführden scheint. Daher verdienen die Erfahrungen der Fälle, welche oben beschrieben worden sind, zur Kenntniss gebracht zu werden. Was die alteren Aerzte über Nierendystopie berichtet haben und was unsere Fälle lehren, sei in Kürze vorgeführt.

Ich habe in der gesammten Literatur über die Lageanomalien der Niere nur sechs Fälle angeführt gefunden, in denen die dystope Niere während des Lebens subjectiv oder objectiv zur Wahrnehmung gekommen wäre, alle, ohne dass die Missbildung erkannt ward. Einer dieser Fälle ist von HohI') beschrieben, welcher eine im Becken liegende linke Niere bei einer 75jährigen Frau fand und dazu berichtet, dass dieselbe $30 \mathrm{Jahr}$ früher bei einer Geburt als Geschwulst im Unterleib gefühlt wurde und bei jeder Wehe vorgetrieben ward. Caudemont ${ }^{2}$ ) erzählt von einem Manne, der an Blasensteinen litt, und bei dem 2 Finger unter dem Nabel eine abgerundete, auf Druck empfindliche Geschwulst gefühlt wurde, die sich nach dem Tode als die vor dom Promontorium gelagerte steinkranke Niere erwies. Durham ${ }^{3}$ ) fand in der Leiche eines Mannes eine tielliegende linke Niere. 5 Jahre vorher wurde dieselbe bei Gelegenheit einer Erkrankung des Betreffenden in Hypogastrium links von der Medianlinie als Geschwulst wahrgenommen, deren Betasten unangenehme Emptindungen hervorrief. Fischel ${ }^{4}$ ) erzählt von einer tiefliegenden Niere, die ein Geburtshinderniss abgab. Sehr sehwere Symptome machte die tiefliegende Niere. in einem Fall, den de Neufville ${ }^{5}$ ) genau beobachtet und dargestellt hat. Es trat

3) Hohl, Meckel's Archiv. 1828.

2) Caudemont, Bull de la Soc. d'anatomie de Paris. 1844.

3) Durbam, Guy's Hosp. Rep. 3. Ser. 6.

b) Fischel, Prager med. Wochenschr. 1885.

5) De Neufville, Archiv für phys. Heilkunde. 10, 1851. 
bei einer 25jährigen Frau innerhalb weniger Tage Oedem beider Beine und bald nachher auch geringer Ascites auf, nach Verlauf von 2 Wochen erfolgte der Tod. Bei der Section zeigten sich die beiden Nieren verwachsen, vor dem Promontorium liegend, sehr blutreich. Durch Compression der Vena cruralis and iliaca externa sinistra war es zur Thrombosirung dieser Gefässe gekommen, der Thrombus hatte sich bis in die Vena cava fortgesetzt und dadurch den Abfluss des Blutes aus der rechten Extremität behindert. De Neufville erklärt das plötzliche Eintreten so schwerer Erscheinungen, nachdem während 25 Jahren keine Krankheitssymptome von dem tiefliegenden Organ ausgegangen waren, durch die Hyperämie der Niere, die im Anschluss an eine Bronchitis und Pleuritis entstanden sei.

In diesem Fall sieht man einmal ein Symptomenbild, das man, wie Lanceraux ausführt, öfters bei Nierendystopie erwarten könnte, nehmlich die Compression der grossen Körpervenen und ihre Folgeerscheinungen. In einer grossen Anzahl der Fälle von Tieflage liegen die Nieren den Vasa iliaca unmittelbar auf, trotzdem habe ich sonst keinen Fall gefunden, bei dem von Druckerscheinungen der Venen für die untere Extremität berichtet wäre. Auch Druck der Nerven bei Lage im kleinen Becken ist nicht beobachtet.

In einem Fall, den Fossati $)$ mittheilt, trat bei einem Arbeiter im Anschluss an ein Trauma ein weich elastischer fluctuirender Tumor in der Unterbauchgegend auf, dessen Inhalt sich bei der Punction als Harn erwies. Nach dem Tode zeigte sich, dass der Tumor einen von Peritonäum und Pseudomembranen abgegrenzten Sack darstellte, der mit dem Nierenbecken der vor dem Promontorium gelagerten Niere zusammenhing. Weiteres habe ich über diesen Fall, von dem mir nur ein Referat vorlag, nicht erfahren können.

Auch unsere Fälle vervollständigen das Symptomenbild, das die tiefliegende Niere hervorbringen kann, nicht. Man könnte geneigt sein, die Verdauungs- und Stuhlbeschwerden, welche in Fall 3 auftraten, auf ein durch die tiefliegende Niere hervorgerufenes Passagehinderniss des Darms zu beziehen. Doch

1) Fossati, Schmidt's Jahrb. 1888. 
hat diese Anuahme weng Wahrscheinlichas, wenn man bedenlat, dass die Niere 30 Jahre lang im Beckenoingang lag, ohne Stublbeschwerden zu machen, und dass letztere eintraten, ohne dass eine pathologische Veränderung der Niere hinzugekommen wäre. Im Zusammenhang mit Vomitus matutinus und den sonstigen Magensymptomen wird die Verstopfung eher als Ausdruok einer alkoholischen Verdauungsstörung zu deuten sein.

Bei so spärlichen und wenig mit einander übereinstimmenden Beobachtungen kann von einem typischen Symptomenbild der Nierendystopie nicht die Rede sein. Es muss vielmehr als typisch bezeichnet werden, dass keine Erscheinungen von der verlagerten Niere ausgehen. Deshalb wird dieselbe auch dem Arzt, der auf sie aufmerksam wird, stets grosse diagnostische Schwierigkeiten bereiten, und es wird selbst in Fällen, wo an oine Nierendystopie gredacht wird, bei der Muthmaassung bleiben. $\mathrm{Zu}$ dem umfangreichen Capitel von den diagnostischen Schwierigkeiten, welche Bauchtumoren verursachen können, liefert, wie unser dritter Fall zeigt, auch die Nierendystopie ihren Beitrag. Aber nicht allein an die diagnostischen Fähigkeiten des Arztes stellt die verlagerte Niere hohe Anforderungen, sondern auch seine operativen Eingriffe können, wie wieder unser dritter Fall darlegt, durch eine solche Anomalie in ausserordentlichem Maasse erschwert and in ihrem Resultate getrübt werden.

Es ist mir, nach den bisher gemachten Veröffentlichungen, kein Fall ausser denen unserer Beobachtung bekannt geworden, bei welchem die tiefiegende Niere zu einem chirurgischen Eingriff Veranlassung gegeben hätte, oder bei einer Operation als störendes Moment hervorgetreten wäre. Allein, wie Eingangs erwähnt, steht zu erwarten, dass der fortgeschrittenen Chirurgie in Zukunft derartige Erfahrungen bevorstehen. Es sind zwei Möglichkeiten in Betracht zu ziehen, bel denen die dystope Niere für den Chirurgen Wichtigkeit erlangen kann. Entweder geht die Indication zur Operation von der Niere selbst aus, oder es handelt sich um jene Fälle, wo Krankheitssymptome fälschlicher Weise auf einen ungewöhnlichen Tumor bezogen werden, ker sich bei der Operation unvermuthet als die Niere erweist. In beiden Eventualitäten hat die Operation ibre Schwierigkeiten und Gefahren. Im letateren Fall sind es hauptsächlich dia- 
gnostische Schwierigkeiten, denn, wie die Operation unseres Falles lehrt, ist der Nachweis, dass solch ein Tumor an der hinteren Bauchwand die Niere ist, keineswegs leicht zu führen während der Operation, und bringt ausserdem Gefahren mit sich. Dass die dystope Niere selbst eine Indication zum Eingreifen geben kann, ist in Hinblick auf Fälle, wie z. B. Fossati einen beschreibt, und im Hinblick auf das gelegentlich auch bei dystoper Niere beobachtete Vorkommen von Hydronephrose und Nierensteinen, nicht ausser Bereich der Möglichkeit.

Dann kann die den Fall complicirende Tieflage der Niere entweder vorher diagnosticirt sein oder erst nach der Eröffnung des Bauches erkannt werden. In beiden Fällen sind die Gefahren und Schwierigkeiten der Operation sebr beträchtliche, dann natürlich besonders, wenn man auf die Tieflage der Niere nicht vorbereitet ist. Die Schwierigkeiten, die sich dem Chirurgen entgegenstellen, liegen in den abnormen anatomischen Verbältnissen begründet. Da kommen zunächst die unregelmässigen Gefässverhältnisse in Betracht, die Mehrzahl und der abnorme Ursprung der Arterien. Der oft nur kurze Verlauf der Arterien und Venen, und somit die enge Beziehung zu den benachbarten grossen Gefässstämmen des Körpers erhöhen die Schwierigkeit und vermehren die Gefahr umfangreicher Blutungen. Ferner wird die Entscheidung der höchst bedeutsamen Frage, ob es sich um eine verwachsene Niere, ob um eine einfach tiefliegende, ob um die einzige und tiefliegende Niere handle, nicht leicht zu treffen sein. Die verwachsene Niere wird wieder je nach dem Grad der Verwachsung eine verschiedene Behandlung indiciren. Ich erwähne hier in Kürze, dass die Durchtrennung der die beiden Nieren am unteren Pol verbindenden Substanzbrücke bei normal gelagerter Hufeisenniere einmal mit Erfolg von Socin ${ }^{1}$ ) ausgeführt worden ist.

In einem anderen Fall, der in der Heidelberger chirurgischen Klinik zur Operation kam und von $\mathrm{Braun}^{2}$ ) beschrieben ist, fand man, beim Versuch eine Pyonephrose zu operiren, dass dieselbe einem Schenkel einer Hufeisenniere angehörte. Es traten so heftige Blutungen aus den mit der Niere verwachsenen grossen

1) Socin, Bruns' Beiträge. 4.

2) Braun, Deutsche med. Wochenschr. 1881.

Archiv f. pathol. Anat. Ba.137. Hft. 2. 
Körpervenen ein, dass die Operation nicht zu Ende geführt werden konnte.

Endlich kann auch das abnorm gelagerte und gebildete Nierenbecken, der abnorm verlaufende Ureter bei Dystopie der Niere eine die Operation erschwerende Complication abgeben.

Kurz, ein operativer Eingriff bei tiefliegender Niere ist in mehr als einer Hinsicht eine dornenvolle Aufgabe und wenn wir Alles, was von praktischen Gesichtspunkten aus über die Nierendystopie gesagt worden ist, zusammenfassen, so bringt diese Anomalie gelegentlich für den Arzt Aufgaben, welche die höchsten Anforderungen an sein Urtheil und Können stellen. In solehen Fällen - mögen es immerhin Ausnahmefälle sein wird die Kenntniss der anatomischen Verhältnisse der Lageanomalie, sowie der über sie gemachten praktischen Erfahrungen von Nutzen sein.

Zum Schlusse sage ich Herrn Geheimrath Arnold für die gütige Ueberweisung dieser Arbeit und seine wohlwollende Unterstützung bei Abfassung derselben meinen wärmsten Dank.

\section{L i t e rat u r.}

(Die mit * bezeichneten Fälle habe ich nur in Referaten eingesehen.)

I. Fälle von Tieflage der linken Niere bei normaler Lage der rechten.

1. Gruber, Wiener medicinische Jahrbücher. 1866. (Alle Fälle der alten Literatur enthaltend.)

2. Gruber, dieses Archiv. Bd. 78.

3. Friedlowsky, Allg. Wiener med. Ztg. 1867.

4. Stern, Inaug.-Dissert. Berlin 1869.

5. Legg Wickham, Transact. of path. Soc. XXVII. 1876.

6. Sehott, Oesterreich. Zeitschr. für prakt. Heilkunde. 1866.

7. Westphal, Berl. klin. Wochensehr. 1890.

8. Lancereaux, L'union médicale. 1880 .

9. "Fossati, Ann. univers. die Med. e Chir. Gingno 1888.

II. Fälle von Tieflage der rechten Niere bei normaler Lage der linken.

1. Drouin, Lieutandi Anat. pract.

2. Treu, Commercium litt. Norimbergiae 1737.

3. Bousquet, Samml. auserlesener Wabrnehmungen. VI. 1762.

4. Varnier, Mémoires de l'Acad. de Paris. 1774. 
5. Chambon de Montaux, Observ. clinic. Paris 1789.

6. Boinet, Archives générales. VII. 1835.

7.* Legg and Omerod, Transact. of path. Soc. XXVII. 1876.

8. Gruber, Mémoires de l'Acad. de St. Pétersbourg. VII. Ser. Tome II. 1860.

9. Gruber, dieses Archiv. Bd. 32 und 68.

10. Gruber, St. Petersburger med. Zeitschr. 1863.

11. Weisbach, Wiener med. Wochenschr. 1867.

12. Stern, Inang.-Dissert. Berlin 1869.

III. Fälle von Tieflage einer Niere bei Feblen der anderen.

1. Weisbach, siebe aben.

2. Watson, Edinb. Journal. 1874.

IV. Tieflage beider Nieren ohne Verwachsung.

1. Martin St. Ange, Annales des sciences naturelles. sér. I. t.XIX.

2. Boyd, Medico-chirurg. transact. London. 2 Ser. Vol.6. 1841.

3. Poirier, Bulletin de la Société d'anatomie de Paris. 1889.

4. Potherat et Morderet, ibid.

V. Gekreuzte Dystopie ohne Verwachsung.

Weisbach, siehe oben.

VI. Fälle von gebreuzter Dystopie mit Verwachsung und von Dystopie beider mit einander verwachsener Nieren.

1. Morgagni (De sedibus et causis morborum. Epist. XLVII) berichtet von einer Anzahl nicht genau besehriebener Fälle von Carolus Stephanus, Andernacas, Cabrolius, Cel Fontanus, Vesalius, Eustacbius.

2. Laube, Ephemerid. nat. curios. ann. IX.

3. Riolanus, Anthropographie.

4. Monginot, Journal des savants. 1678.

5. Botallus, Observ. anat. I.

6. Panthot, Journal des savants. 1681.

7. Sandifort, Museum anat. Lugd. Vol. I. - Observ. anat. path. Lib. III.

8. Du Vivier, Mém. de l'Acad. de Paris. 1730.

9. Balley, Auserles. Wahrnehmungen. Strassburg 1764 .

10. Haller, Opuse. path. 1755.

11. Gebhard, Advers. med. Basileae 1777.

12. Kaltschmidt, Diss. de uno rene in cadavere invento. Jenae 1755

13. Pan arolus, Jatrologism. observ. Pentec.

14.* Mondoni, Novi comment. acad. scient. Bonn 1839.

15.* Sandwith, Prov. med. Journal. 1844.

16.* Reid, Monthley Journal. 1843.

17.* Penada, Saggio d'osserv. t.I.

18. Godard, Comptes rendus de la Soc. de biologie. 1855 . 
19."Goux, Gazerte de Paris. 1856.

20. De Nentville, Archiv f. phys. Heilkunde. 1851.

21. Gruber, dieses Archiv. Bd. 10?.

22.* Braxton Hicks, Transact. of obstetr. Soc. London 1879.

23. Bachhammer, Archiv f. Anat. u. Entw. 1879.

24. Weisbach, Wiener med. Wochenschr. 1867.

25. Stocquart, dieses Arehiv. Bd. 78.

26. Kundrat, Wien. med. Wochenschr. 1886.

27. Birmingham, Dublin fournal. 1890.

28. Kruse, Inang.-Diss. Greifswald 1890.

Ausführliche Behanding und Literaturangabe der Lageanomalien ler Niere findet man ausserdem in den Handbüchern von: Ziegler, Orth, Klebs, Forster, Birch-Hirschfeld, Mectel, Voigtel, und bei Rayer, Traité des maladies des reins.

\title{
XII.
}

\section{Klinische Basis zil meiner Theorie) über die parenchymatöse Nephritis und ein Beitrag zur Aetiologie des rumden Magengeschwiires.}

\author{
Von Dr. Alexandre Favre, \\ Yrof. ag. de Médeeine légale à l'Académie de Neuchâtel.
}

I.

In einer Reihe von Abhandlungen ${ }^{2}$ ) war es mir gelungen, eine ganze Anzahl von Krankheitserscheinungen ätiologisch zu begründen. Die Schwangerschaftsniere erwies sich als eine theils infectiose, theils physikalische Nephritis. Ebenso die Puerperaleklampsie war es möglich in den meisten Fällen ${ }^{3}$ ) als eine Ptomaïnämie hinzustellen.

1) Vortrag im Congress westschweizerischer Aerzte, gehalten vom Verf. am 19. Oct. 1893 in Lausanne. Revue médicale de la Suisse romande. XI. 1893. Genêtye.

2) Alex. Favre, dieses Arehiv. Bd. 123, 124, 127, 129.

3) Wenn Dhürssen die Behauptung aufstellt, die Eklampsie sei in den meisten Fällen auf eine Intoxication des Blutes zurück $z u$ führen durch Retention von Kreatin und Kreatinin, so ist dieselbe als rein speculative Hypothese aufzufassen. Vom Krankenbette sind dabei keine positiven Daten entrommen. 